\title{
Article
}

\section{Hydrocode investigations of Terminal Astroballistics problems during the hypothetical Future Planetary Defense System's Space Mission.}

\author{
Maciej Mroczkowski ${ }^{1,}$, Stanislaw Kachel ${ }^{2 *}$ and Adam Kozakiewicz ${ }^{2}$ \\ 1 Institute of Optoelectronics, Military University of Technology, 00-908 Warszawa, Poland; \\ maciej.mroczkowski@wat.edu.pl \\ 2 Faculty of Mechatronics, Armament and Aerospace, Institute of Aviation Technology, Military University \\ of Technology, 00-908 Warszawa, Poland; stanislaw.kachel@wat.edu.pl, adam.kozakiewicz@wat.edu.pl \\ * Correspondence: stanislaw.kachel@wat.edu.pl; Tel.: +48-261-839-170
}

Received: date; Accepted: date; Published: date

\begin{abstract}
The article is devoted to the preliminary concept of the Future Planetary Defense System (FPDS), emphasizing Astroballistics. This paper is intended to support international efforts to improve the planetary security of the Earth. The work covers three areas of knowledge: Astronautics, Astrodynamics, and Astroballistics. The most important part of the presented article is dynamic, contact combat modeling against small, deformable celestial bodies. For these purposes, the original, proprietary hydrocode of the Free Particle Method (HEFPM-G) with gravity was used. The main aim of combat is to redirect the Potentially Hazardous Objects (PHOs) to orbits safe for the Earth or destroy them. This concept's first task is to find, prepare and use dynamic threedimensional models of celestial bodies' motion and spacecraft or human-crewed spaceships in the Solar System's relativistic frame. The second task is to prepare the FPDS' architecture and computer simulation space missions' initial concepts in the internal part of the solar system. The third and main task covers simulating, using hydrocodes, selected methods of fighting $100 \mathrm{~m}$ and $140 \mathrm{~m}$ diameter asteroids. The order of the article is as follows. The first part of the article presents an architecture and FPDS' modus operandi. Preliminary design and development of FPDS' space missions, including navigation, mission dynamics simulation, is prepared using an open-source space mission analysis and design tool. E.g., Asterank and Trajectory Browser or GMAT are presented in the second part. The third part of the article is devoted to computer hydrocodes (HEFPM-G) and the modeling and simulation of asteroid-asteroid collision, laser radiation effects on an asteroid, and FPDS spacecraft's warhead contact interaction on the small celestial body. The authors formulated the Main Planetary Defense Problem (MPDP) in this paper. The proposition of this problem solving has been realized by preparing its concepts, architecture, and modus operandi of the FPDS mission. Finally, a series of realistic simulations were made using hydrocode to deflect or destroy dangerous asteroids. The summary and conclusions can be found in the fourth part of the article.
\end{abstract}

Keywords: astronautics; astrodynamics; terminal astroballistics; the main planetary defense problem; hydrocode; warheads; impulsive dynamic interactions on the asteroids; modeling and simulation; sensors; network-centric system architecture; space missions; navigation; planetary defense modeling; synchronization; atomic and pulsar time; autonomy; space; relativistic metrology;

\section{Introduction}

In the paper, an original proper Hydrocode of Free Particles Methods (HEFPM-G) with gravitation [1] is applied to simulate, more precisely, the Terminal Astroballistics problems with three 
methods of contact deflection of virtual Potential Hazardous Asteroids (PHAs). PHAs are the part of small celestial bodies in the space of the Solar System. The issue of collisions of small celestial bodies has been exploited since the early 1950s. Some collisions can generate small solar system bodies (SSSBs), which can become dangerous as PHA and eventually hit the Earth. The most interesting from the future defense space system (FPDS) point of view is cosmic collisions in the Inner Solar System space area. The greatest probability of their occurrence concerns the space close to the ecliptic plane.

For this reason, it is necessary to systematically use the Astronautics and Astrodynamics methods for motion modeling and simulations in this area of Solar System space. For the terminal astroballistics' simulation of small celestial bodies' contact interactions, "typical" problems were selected. They could occur anywhere in the inner part of the Solar System space during future Defense Space Mission. This paper's primary goal is to simulate, as realistically as possible, the impulse interactions on small, dangerous space bodies. This is the decisive and final phase of the original project of the FPDS Space Mission. The order of issues discussed in this publication results from the imposed chronology of space system preparation primary ideas and space missions implementation rules [2,3]. The necessary participation of astronauts in FPDS bases on Earth, the Moon, and Mars and manned space vehicles introduces the need to model the astronautics problems of the human-crewed spaceship in the near future.

\subsection{Mathematical-physical model of Solar System dynamics in barycenter relativistic frame and Future Planetary Defense System (FPDS) network-centric architecture.}

The first part of this article gives the mathematical-physical models basic for the Solar System dynamics in relativistic frames [4,5]. After that, an abridged version of the description of the FPDS network-centric architecture (FPDS is distributed in the inner part of the Solar System) is presented [6].

\subsection{GMAT's example of FPDS mission to Potentially Hazardous Object (PHO).}

In the second part of the article, a professional open-source computer system "developed by a team composed of NASA, private industry, and a range of academic, public and private contributors" the General Mission Analysis Tool (GMAT) [7] was used. The GMAT made it possible to obtain results from simulating astrodynamic problems and navigating to potentially dangerous small celestial bodies. In the simplest case this made it possible to effectively navigate an autonomous [8] or manned spacecraft and perform planetary maneuvers to reach the close vicinity of a dangerous asteroid. Under such conditions, it is possible to obtain spacecraft warheads - asteroid contact interactions in an individual action. In the paper, asteroids were selected for analyzes with a diameter of 100 meters (very common, as suggested by Mark Boslough [9]) and a diameter of 140 meters (according to George E. Brown, Jr. Near-Earth Object Survey Act of 2005 [10]), because they are a large risk to the human population. However, there are still problems with recognition, spatial situational awareness, and decision-making simulations in more complex cases generated in the solar system's deep space. The sources of the problems are asteroid and comet collisions, gravitational interactions, and threats from interstellar intruders. Relationship modeling in the FPDS [11] networkcentric architecture is only partially possible inside the GMAT. This should be done using MATLAB's web tools or software written in Python, adapted to the GMAT interface. Finally, after improvements, in the fully network-centric, autonomous [10] and multi-spacecraft FPDS' missions, the technology of asteroid deflection or destruction is ultimately expected to protect the Earth from impact. The necessary definitions, an example FPDS space mission scenario, and the initial-boundary conditions of selected simulations of spacecraft dynamics and small solar system bodies (SSSB) were also discussed. This section introduces a new class of astronomical objects from a planetary defense perspective: the interstellar object $[12,13]$.

1.3 Hydrocode modeling and simulations of Potentially Hazardous Asteroids (PHAs) deflection or destruction, and the Main Planetary Defense Problem. 
The third part presents basic models of the dynamics of solid small celestial bodies collisions in the presence of gravity, as well as models of impulse interactions on PHAs with the use of profiled explosives and laser radiation. The purpose of this computer modeling is to find such parameters of the spacecraft's warhead operations that will allow to solve the Main Planetary Defense Problem (MPDP - see point 1.1) by deflection or even destruction of a certain class of dangerous asteroids (100 meters or 140 meters in diameter) on a collision course with Earth. Destruction of asteroids should be done by crushing or spalling and dispersion. The work, at this stage, assumes deflection by explosives or impact or laser interactions, but the collection of available methods will be expanded as the project develops. The problem of the destruction and dispersion of asteroids and comets will be discussed in more detail, including nuclear technologies, in subsequent articles. The simulations were performed using updated proper Hydrocode of the Free Particles Method with Gravitation prepared for solving the Shock-Wave Physics problems. This hydrocode has been comprehensively verified, calibrated, and positively validated during the modeling of many cases of deformable celestial body collisions, as shown in publications [1] and [17-24]. As spacecraft warhead operations are most effective at close range, the final part of the Future Planetary Defense System (FPDS) mission will start at the end of precision deep space navigation phase. To maintain the responsiveness of the defense system, three-stage armed space vehicles capable of independent operations are planned (see point 1.2). Depending on how the situation develops, it will be possible to prepare 3D maneuver in space flight, including fly-by, rendezvous, or approach a collision straight from the retrograde orbit. The solving of the main problem of planetary defense results from building up gradually a proper system. Basic requirements include a high autonomy level, with the sensors to navigate, recognize and track, and the warheads' actuators to intercept and redirect or destroy "almost all" PHO. PHO means all asteroids and comets that have sufficient geometric, material, and dynamical parameters to threaten the Earth's population. The issue of sensor subsystems is closely related to the space metrology problems of the spacecraft atomic and pulsar time [3]. The global synchronization between the spacecraft and local synchronization on the spacecraft board are very important in the Deep Space of the Solar System. The primary system, applied to the Solar System scale, is a set of nodes and connections between them. A proper architecture model of the distributed network-centric planetary defense system, where the nodes are autonomous spacecraft, with autonomous physical sensors on board, is proposed. Laser communications connect the nodes. In the presence of the Sun, planets, and small celestial bodies, the relativistic theories for a physical description of dynamical phenomena are applied. As recommended by the IAU a Post-Newtonian equation of motion model in a relativistic coordinate system is presented. The proposed accuracy of the measurement sensor reaches the level of space quantum metrology tools (see section 2.2 - SI 2018).

\section{Initial remarks on Paradigm of Research in Science using to solve the Main Planetary Defense Problem (MPDP). Architecture and the final configuration of FPDS mission geometry}

This article is a development of a work on the problems of the Future Planetary Defense System (FPDS) modeling [4]. The focus has been shifted from the synchronization and coordination (with the correlation) problems of autonomous sensor systems to local hydrocode interactions modeling. At an early conceptual stage, it is most important to make effective use of computer experiments. In this way, modern or future technologies of highly effective combat systems should be found and quantified. It is known, that the best is short-range action or even contact interaction using impulsive methods [Holsapple] to disrupt and disperse PHO or deflect it. This means that the FPDS architecture (see point 2.3) that guarantees the possibility of early and contact action on PHO will be of fundamental importance. Architecture is the structure of components, their relationships, and the principles and guidelines governing their design and evolution over time [DoD Architecture Framework Ver.1.0, based on IEEE STD 610.12]. FPDS architecture is an evolution of C4ISR Architecture Framework toward NCOW, SSA and ODDA from Operational View (OV), System View 
(SV) and Technical Standards View (TV). However, the FPDS like a whole, large system in inner Solar System space, must work in a strictly defined sequence of steps. It needs synchronization.

To do this, architecture geometry modeling starts with relationship between $\mathrm{A}, \mathrm{B}, \mathrm{C}, \ldots$, and $\mathrm{Z}$ clocks synchronization and coordination. Correlations should be understood in the sense of the Poincare-Einstein convention on a closed road. This issue is important for FPDS clocks in orbit around the Sun. But even in the Earth layer of FPDS, the amount of necessary relativistic corrections for FPDS' spacecraft clocks, can be estimated e.g. from the Global Positioning System (GPS) system satellites clocks in orbit around the Earth. The next step is about FPDS synchronization events modeling, as a coordination of the joint operation of the entire future planetary defense system problem.

The paper refer to some relativistic models: a) in the context of Special Relativity (SR) - in the case of synchronization and correlation, and data transmission exchange, by using electromagnetic wave propagation and propagation of laser beams as a photon stream. The latter also applies to the use of a laser to destroy asteroids; b) within the framework of approximations of General Relativity (GR) - in the case of massive bodies motion.

In this work, the extended paradigm of research in science is used (see Fig. 1). It specifies the interdependence between theory, observation, and experiment, which together form the best mathematical and physical models of a computer experiment capable of simulating future events. The paradigm is applied through first pillar: analysis, selection, and adaptation of the appropriate Theory. In the second pillar the Experiments and the observations are conducted. It allows the verification of the selected theory. In the third pillar - the Computational experiment in Virtual Laboratory, which is about simulations of physics in the Virtual Reality (VR) - the mathematical, physical, and numerical models are prepared. After writing and running the programs for simulation and visualization of calculations it is possible to compare results from VR with reality.

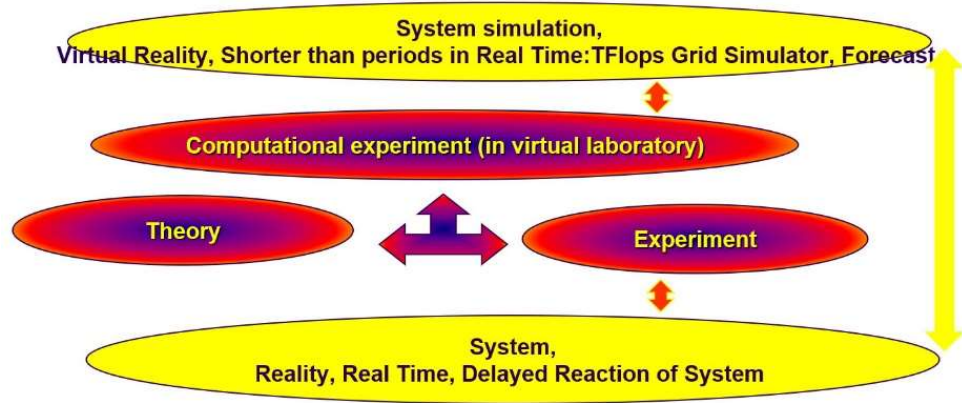

Figure 1. Paradigm of Research in Science (in red) with ideas of FPDS simulation (in yellow).

The sequence of actions presented in the paper with the use of precise computational experiments is the correct conclusion from the scientific paradigm.

2.1 The Main Planetary Defense Problem (MPDP) of the Future Planetary Defense System (FPDS).

As mentioned above, the solving of the real Main Planetary Defense Problem (MPDP) mean that "almost all" Potentially Hazardous Objects (PHOs) will be redirected or destroyed. The modeling and simulating in virtual reality the real dynamical processes in Solar System is very difficult. In this case, very high accuracy is required to guide warheads towards asteroids. Additional problems are connected with modeling of a proper FPDS with high autonomy level spacecraft, and theirs subsystems to recognize, track, intercept and redirect or destroy "almost all" asteroids and comets that have sufficient parameters to threaten the Earth's population. Real MPDP ends with the 
destruction of the Earth-threatening object. In VR, new measures will have to be developed to assess the degree of destruction of the attacked asteroid. Simulating the processes taking place inside the attacked asteroid is very difficult to model and interpret, so now more emphasis will be placed on it. The initial scheme of short-range final stage of FPDS activity is shown below on Figure 2, but the rest of the endeavors will be connected with computer modeling and simulations of virtual Main Planetary Defense Problem.

\subsection{The configuration of an autonomous space vehicle for recognition, tracking, and destruction of asteroids.}

The planetary system's functionality is tied to the three-phase concept of combating a potentially hazardous object (PHO) that threatens the Earth. In this paper, a potentially hazardous asteroid (PHA) was selected to describe the Earth defense mission's final. The first phase of the final includes reconnaissance, research and analysis, and computer simulations of the attack. The second is an optimal attack. The third phase of the finals consists of assessing the effects and possible repetition of the attack.

For this reason, the spacecraft has three stages; each spacecraft's stage is autonomous and performs one attack phase (see the point).

The first phase's details will concern remote recognition of the asteroid's shape and composition using image spectrometry and autonomous sensors for precise shape scanning and surface morphology. Further sub-phases will determine the mean density, mass, sampling to determine the local porosity and possibly the strength of the mixtures of components of the asteroid material. The collected samples will be used to study the dynamic properties of materials. On this basis, a dynamic, virtual mathematical-physical model of an asteroid will be prepared as a correctly posed initial- boundary value problem. The calculated effects, as the response to dynamic loading, allow the optimization of attack. It will happen after launching a computer program related to the model and carrying out multi-variant simulations of the operation of available warheads and, for example, laser systems.

The attack will be launched after the astronaut's decision in the command and control loop.

Details of the attack carried out in the second phase of asteroid control will be collected online from the autonomous network-centric sensors installed in the first phase on the surface and below the asteroid's surface and in orbits around it. During recording, data will be used to evaluate the processes taking place in real-time. The network-centric sensor system will include numerous radio, radar, and optoelectronic sensors operating in various parts of the electromagnetic wave spectrum, such as VIS, IR, UV. Local sensors for in-situ measurements of displacements, velocities and accelerations, pressure and temperature sensors, and special seismic sensors will assess on-line attack's effects. It will be possible, almost in real-time, by comparing the measured values with the simulation results. Dust generated by the attack will not be an obstacle.

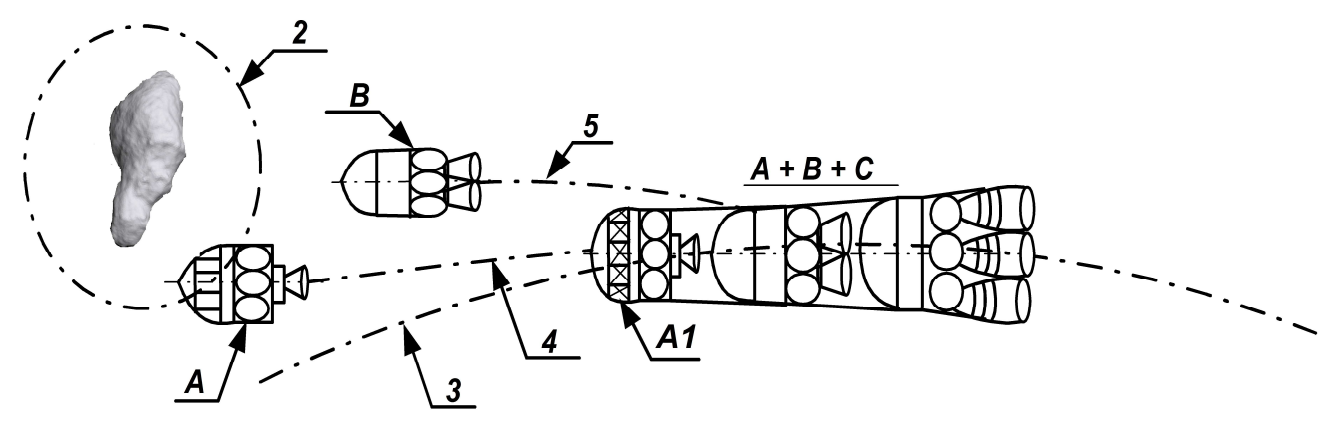

Figure 2. The short-range final part of FPDS activity with use of single Autonomous Spacecraft. This mission is finished with three stages of Autonomous Spacecraft using specific loops of activity. For our purposes it is possible to extend the OODA loop developed by Colonel John Boyd, which is the 
cycle: observe-orient-decide-act, by the cycle observe-orient-decide-act-check and repeat the loop OODA. The Potentially Hazardous Object (PHO), here it is the Near-Earth Asteroid (NEA), is on the collision course with the Earth. Asteroid was detected and tracking before by the A1 - MEGA SENSOR part of A sub-spacecraft. It is the third stage of Autonomous Spacecraft for detection and observation the close space of NEA orbiting during mission action. Pico- or femto-space probes for surface landing and local NEA research may also be used. NEA is attacked by using the B - MEGA SPACEWARHEAD actuator subsystem as second stage of Autonomous Spacecraft for destruction the hazardous NEA. The C "mother", as the first stage of Autonomous Spacecraft, observing the mission results, secures this action of the FPDS. Spacecraft Stage C, or "mother", may repeat the attack if the second stage $\mathrm{B}$ attack fails. Here $\mathrm{A}+\mathrm{B}+\mathrm{C}$ mean the complete Autonomous Spacecraft during approach phase to NEA.

\subsection{Motion and General Theory of Relativity. Quantum metrology.}

Mathematical and physical dynamic modeling of the Future Planetary Defense System (FPDS) is a challenge. Modeling begins with selecting an appropriate physical theory that must consider the dynamics of network-centric autonomous spacecraft. In this case, the Resolutions of the XXX IAU General Assembly of 2018 formally force the modeling of motion in relativistic coordinate systems. Simultaneously, they caused the use of relativistic models of body dynamics, resulting from Einstein's General Theory of Relativity, in the so-called post-Newtonian approximation [4,5]. Besides, large numbers of autonomous sensor systems and optoelectronic effectors are installed onboard spacecraft. Their operation should be modeled, considering the relativistic models of electromagnetic wave and photon flux propagation resulting from Einstein's General and Special Theories of Relativity. It is imperative when their trajectories are close to the Sun or Jupiter. This kind of modeling is a new task compared to current modeling practice.

Additionally, in the case of autonomous space navigation, modeling requires the latest developments in space technologies at the level of quantum metrology. Many observations and research results from the area of interest, for obvious reasons, are still subject to many uncertainties. The next step is selecting relativistic coordinate systems, barycentric for the Solar System in its entirety and geocentric for the Earth. They allow setting the correct initial and boundary value problems (IVP and BVP). IVP and BVP are necessary for the proper modeling and simulation of the sequence of events that interests us. Interpretation difficulties require a more detailed discussion on modeling relativistic problems in the Solar System.

The principle of equivalence, which is also described in the General and Special Theories of Relativity, applies to all physical laws. The principle states that the local properties of curved space in relativity should be indistinguishable from the properties of flat space-time in special relativity [4]. It is the path to the mathematical-physical algorithms of relativistic motion models. The concept of motion does not appear in relativity. But, by investigating the exact solutions of Einstein's nonlinear equations, Einstein, with Infeld and Hoffmann (EIH) [4], proposed a methodology for a linear approximation of Einstein's equations for the relativistic problem of two-body interaction in a weak gravitational field and at a low speed (concerning the speed of light). Infeld and Plebański summarized Einstein's approach in the book "Motion and Relativity" [5] to solve the relativistic motion of N-bodies.

3. Initial assumptions of the Future Planetary Defense System: Geometry, Reference Systems, Sensors and Architecture Framework in Space Environment

\subsection{Geometry and Reference Systems}

The Future Planetary Defense System (FPDS) is intended to be an active multi-spherical layered system. The first layer of defense, the most distant, is a sphere with a radius $r=1.25 \mathrm{AU}$ (covering a 
full solid angle of $4 \pi$ steradians), with the center near the Sun in the relativistic Barycentric Celestial Reference System (BCRS). This part of the FPDS works against hazardous celestial objects using four rings of spacecraft covering the sphere. Dangerous objects can be Small Solar System Bodies (SSSBs), which are from the Main Asteroid Belt or Deep Space of the Solar System, or Interstellar Bodies (1I,2I) $[12,13]$. BRCS was generated as part of the application of general relativity, using the method of linear post-Newtonian approximation, with the center in the barycenter of the Solar System. Four rings of FPDS spacecraft were located on the mentioned sphere's surface, each lying on a separate plane. It has been ignored the influence of the Kozai mechanism on asteroids and spacecraft. Each subsequent plane is tilted from+ the ecliptic plane by an additional 45 degrees of ecliptic latitude.

The authors assume that the second layer of defense, the medium range, will be a sphere with a radius of $r=900,000 \mathrm{~km}$. It is close to the boundary but inside the Earth's Gravity Influence Sphere (SOI), with the center in the relativistic geocentric celestial frame of reference (GCRS). As for the first spherical layer, it will consist of four space vehicle rings. It will work mainly against Near Earth Objects (NEOs), namely Near-Earth Asteroids (NEAs) and Near-Earth Comets (NECs). The most dangerous part of the NEAs is a class of Potentially Hazardous Asteroids (PHAs). The third spherical layer of protection, at very short distances, should be a system stationed on the Earth's surface. This system will use the already operational and planned subsystems for the near-Earth Space Situational Awareness (SSA). This system, based on a network of large aircraft-rocket airports and systems for launching rockets from Air-launch to orbit, such as Stratolaunch System aircraft, allows for launching rockets with take-off weights of up to 230,000 kg. The FPDS system supported by a network of bases with human crews on Earth, the Moon, and Mars will have the astronauts in the control loop. In the area of interest of FPDS, there should appear a ring of space probes, in the ecliptic plane, between Mercury and Venus warning about events potentially dangerous for Earth, such as Coronal Mass Ejections (CMEs) from the Sun's surface. Planetary defense systems must be modeled in the Solar System's Space Environment with specific Space Weather, including solar wind, solar flares, CMEs, and various radiation types. These Space Weather events can be a source of serious disturbance to synchronization processes. Among complicated numerical modeling techniques, can be found, e.g., computational magnetohydrodynamics (MHD).

\subsection{Proper time, Atomic and Pulsar Clocks, SI of 2018, Autonomic Spacecrafts, Sensors and Syn- chronization}

The issue of sensors in communications subsystems, such as radio frequency (RF) NASA Jet Propulsion Laboratory (JPL) Deep Space Network or laser communications in space, is critically dependent on the synchronization of their elements. The latter requires the operation of precise distance and angular tracking and positioning of the sensors onto the next spacecraft's laser transceiver, to establish and maintain communication, and high data rate transmission. Since the position and speed must be very accurately known, an autonomous deep space navigation system requires an on-board atomic clock, such as NASA's JPL Deep Space atomic clock. With high stability at the $10^{-15}$ level, this atomic clock must be synchronized and coordinated with a pulsar clock, such as the Polish pulsar clock from Gdansk [3], with stability at the $10^{-18}$ level. All the sensors mentioned above, including their relativistic corrections, must be at the level of precision of Quantum Metrology Standards (SI of 2018). During the modeling of the planetary defense chain's components, some problems related to relativistic phenomena appeared on the Solar System scale, including synchronization processes. This work is an attempt to describe them too.

\subsection{Architecture Framework of FPDS and the DODAF - Department of Defense Architecture Framework}

The term "planetary defense" encourages the use of the achievements of the military theory of Network-Centric Operations and Warfare (NCW) [9]. Within this framework, it is possible to correctly introduce the problems of collecting, transforming, and integrating data from a multi-level, 
hierarchical network of sensors located on spacecraft and distributed in space. In the next step, it is possible to process data into useful information that will be used to build knowledge of Space Situational Awareness (SSA). Ultimately, the people on the Earth and astronauts in space, present in the feedback loop, will be able to decide to run command chains to defend Earth against the impacts of Small Solar System Bodies (SSSBs) or Interstellar Bodies (ISs) with the use of adequate actuators. A top-down methodology incorporating modified Marian Mazur models of the "Cybernetic Theory of Autonomous Systems" [10] was applied. This theory includes the analysis of cognitive problems in conjunction with decision-making problems. Because of a proposition to localizing an autonomous spacecraft system as a top system of sensors in the Deep Space of the Solar System, it is necessary to use relativistic space metrology tools when the SI of 2018 is already in force. Simple, this is necessary because of the universal relativistic coordinate system of the solar barycenter. The IAU resolutions require (starting from 1 January 2019) the use of General Relativity Theory to describe the Space of the Solar System and even local space around the Earth in the weak field limit of the Post-Newtonian equations of "slow" motion (PN). But what does this mean for the clock synchronization subsystems, the entire planetary defense system, and its sensors? It implies very accurate and stable time signals from atomic and pulsar clocks and synchronization in the Poincare-Einstein convention. Finally, it gives the coordination of the joint operation of FPDS within the framework of Special and General Relativity.

The main risk to our civilization comes from Small Solar System Bodies (SSSBs), with the specification of dangerous SSSB diameter, after Mark Boslough's analysis [11], at $100 \mathrm{~m}$ and even below. Due to a large number of small Solar System Bodies (SSSB) with diameters less than and equal to $100 \mathrm{~m}$, the main problem of the Earth's planetary defense becomes complicated to solve. There are additional requirements for the FPDS architecture for clocks and sensors.

At the initial stage of building the computer concept of planetary defense (FPDS), it has been introduced the system architecture issue as the concept of FPDS Architecture Framework modeling based on the ideas of the Department of Defense Architecture Framework (DoDAF v.2.2 and MODAF v.1.2 and NAFv.4) [9]. The process of even limited modeling of FPDS activities to support planetary defense activities and change management during routine service and crisis begins after the definition of the FPDS-Architecture Framework (FPDS-AF). When designing architectural, geometrical, physical, and information mechanisms of the network-centric planetary defense system in space missions, the problem rely on the fundamental definition of our lowest level in the hierarchy, i.e., the autonomous subsystem model according to Mazur's description [10]. It is necessary to emphasize (a) the role of sensors in the information channel for data collection and exchange, telemetry, and system synchronization, and (b) the role of actuators in the energy channel and the collection, generation, and accumulation of energy, and finally (c) the role of the local center of command and control with supercomputer support, and with the appropriate relational database of the Big Data class for mission co-management and Space Situational Awareness (SSA) creation. FPDS-Architecture Framework plays an infrastructure resource of organizational, financial, material, and system tools to prepare and build new concepts of planetary defense capability. It is also used for assessment, from an operational, technical, and systemic point of view, in a mode analogous to Network-Centric Operations and Warfare (NCW). It generates an analysis of the state of Space Situational Awareness (SSA) and future activities that create or restore infrastructure resources. Briefly summarizing, the data flow from FPDS-Architecture Framework allows us to use updated knowledge for long-term management and control of future operations during planetary defense missions.

The first task in our concept's computer modeling is to prepare mathematical-physical, and numerical models, both classic and relativistic. After that, we must simulate key physical processes accompanying emerging threats and combat these threats using massive resources of directed energy, remembering that the effectiveness of planetary defense depends on changing the SSSB's momentum, for example, during collision momentum exchange. 
The second task is to resolve the problems of integration of subsystems and connections between them. The next steps include: identification and modeling the new system capabilities, then formulation software requirements, analyzing costs, and preparing the model of financial service for the mission by estimating the number of subsystems needed to implement the mission.

The third task is to identify, analyze, model, and simulate an autonomous subsystem of sensor interconnected networks using quantum space metrology parameters with relativistic corrections.

The highly energy-demanding physical planetary defense processes coincide with information processes, such as decision-making processes, which have minimal energy consumption. All processes referred to here are "encapsulated" in various categories of extensively distributed sensor networks. These networks must work and be synchronized (a common problem of special relativity theory), considering relativistic corrections for the extremely precise (almost exact) quantum space metrology tools. How to carry this out is a central problem raised in this paper.

\subsection{Future Planetary Defense System closer to the Main Asteroid Belt}

FPDS closer to the Main Asteroid Belt means the perimeter of the system equal to 1.25 AU. Early analysis of threats to Earth from incoming asteroids and comets treated them as single, unique events. The focus was on single space missions, which included the issues of the launch phase, precise navigation, and the arrival of a space vehicle equipped with the appropriate set of actuators (e.g., classic warheads, nuclear warheads, or a super-laser with extraordinary energy and power) directed to the target. It was enough for active interactions with "small" celestial bodies directly threatening the Earth.

An infrared telescope, the Wide-field Infrared Survey Explorer (WISE), has been operating in space for the past ten years (2009-2019) [13]. WISE has discovered 19 new comets and over 33,500 new asteroids, including 120 NEOs relatively close to Earth. In the final phase of the WISE mission, NASA started a new mission called Near-Earth Objects WISE (NEOWISE), which has observed 153,726 Solar System objects. Before hibernation, this spacecraft delivered characterizations of 158,000 minor planets, including more than 35,000 newly discovered objects. Started in December 2013, the post-hibernation NEOWISE mission was anticipated to discover 150 previously unknown near-Earth objects and to learn more about the characteristics of 2,000 known asteroids. NEOWISE's automated detection software, WISE Moving Object Pipeline Subsystem (WMOPS), detected only a few objects smaller than $100 \mathrm{~m}$ in diameter because doing so requires five or more detections. The average albedo of asteroids larger than 100 meters discovered by NEOWISE was 0.14 [14]. Research from WISE/NEOWISE missions involving the search for Small Solar System Bodies using IR sensors revealed the correct scale of small bodies' threats (see Figure 3) [15]. It prompted us to propose research and implement a new future planetary defense system (FPDS) closer to the Main Asteroid Belt.

Step by step, scientific and research missions that successfully navigated into the Solar System's more in-depth and deeper areas were beneficial for this approach. The initial question then arose concerning assessing the "real effects" of such interactions: Is there even a slight possibility of preventing a small celestial body's catastrophic collision with the Earth? Early answers were somewhat optimistic, and a change in the linear velocity component with Delta- $\mathrm{V}=1 \mathrm{~cm} / \mathrm{s}$ of the known asteroid 99942 Apophis should be enough [Sitarski]. But that was only the beginning of the necessary actions.

In the planetary defense case, the team started in the early 1990s [16-19], preparing the hydrocodes of high energy density physics for cosmic bodies for high-velocity collision modeling [20,21]. For several decades, many cases of catastrophic space impacts and collisions have been modeled in this field, with the hydrocodes HEFPM-G with gravity. Many visualizations and computer movies with additional tools helped analyze and understand the processes and effects of collisions [16]. These results were sufficiently positive to start planetary defense system modeling. It 
meant that there were opportunities to change the path or even destroy specific categories of asteroids and comets [Sitarski]. For this reason, we are now able to show the qualitative and quantitative effects of dynamic impacts on a selected class of SSSBs, and even out-of-solar system asteroids (e.g., 1I/'Oumuamua) or comets (e.g., 2I/Borisov).

Our strikes, i.e., planned collisions, are contact strikes, so the spacecraft's precise navigation is critical. It requires the correct selection and construction of several subsystems in which we carry out the necessary sequences of activities to accomplish the mission.

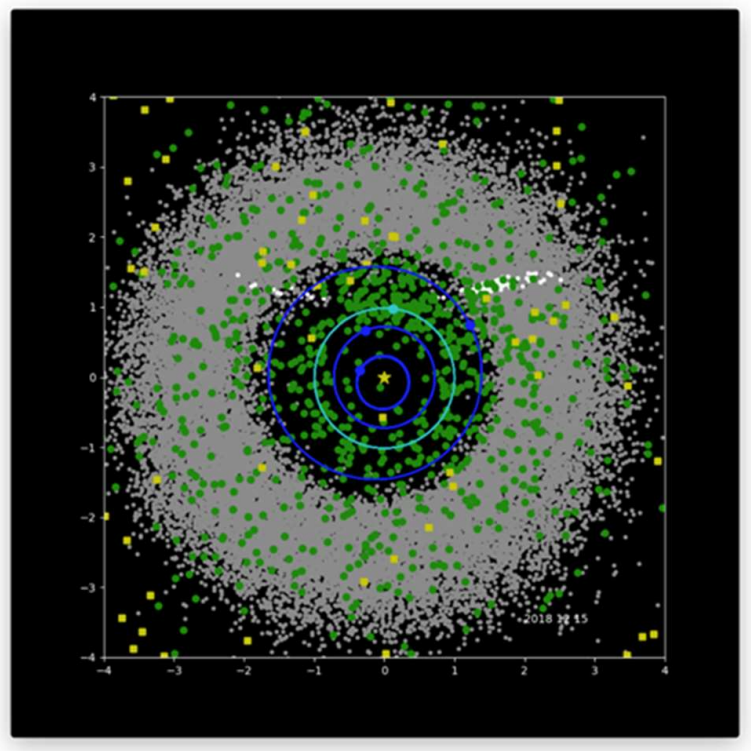

Figure 3. Top-down view of the Solar System showing the position, on 15 December 2018, of all the Main Belt asteroids (grey circles), Near-Earth asteroids (green circles), and comets (yellow squares) detected by the near-earth objects Wide-field Infrared Survey Explorer (NEOWISE) during the first five years of the Reactivation survey. The white circles show the objects detected during the final week of year five. The blue circles and points indicate the orbits and locations of Mercury, Venus, and Mars. The Earth and its orbit are in cyan [15].

\subsection{Precision of navigation}

There are many problems with precision. The first class is associated with a space metrology measuring system for determining the position and velocity of a spacecraft in the General Relativity frame, where the quantum systems of laser radars must work with space metrological precision.

The second class is associated with the synchronization processes and data transmission, which is possible only using Special Relativity Theory. Formally, the synchronization process can occur in space, in which the Special Theory of Relativity is in force, along straight lines, along with closed tracks back and forth. Synchronization cannot take place along beams of photons running near large masses, for example, to the Sun or Jupiter, which is a problem related to bending light beams in gravitational fields.

It seems, however, that data transmission can take place in beams of light photons passing "close" to large masses. Then the essence of data transmission is the flight of a photon bundle, where we reconstruct the states of the photons located in the bundle with each other, without worrying about the time of flight.

The third one is associated with the accurate, relativistic model of the Solar System's motion, the fundamental problem of General Relativity Theory. 
The fourth one is related to the construction of a precise relativistic model of spacecraft motion.

Suppose navigation with relativistic precision leads close to the target, after that to the collision mission. In that case, the decision chain and "fire chain" sequence begins, forming a planetary defense chain and leading to the selected form of interaction with the Small Solar System Body. An example of a collision mission was "Deep Impact." Interestingly, Deep Impact used the autonomous optical navigation (AutoNav) software system to guide the Impactor spacecraft to intercept the nucleus of Tempel 1 at a well-illuminated location, visible from the Flyby. The Flyby spacecraft used similar software to determine its comet-relative trajectory and to provide the attitude determination and control system (ADCS) with the relative position information necessary to point the High-Resolution Imager (HRI) and Medium Resolution Imager (MRI) instruments at the impact site during the encounter [17].

\subsection{Top-down methodology of C4ISTAR to obtain network-centric capabilities with optimal geometry, functionality and autonomy with decision-making model}

Years ago, Norbert Wiener emphasized that we must first have "know-what" before we tackle "know-how," i.e., the technological problem. It suggests giving preference to a top-down methodology in the first research phase of the Future Planetary Defense System (FPDS) computer design. The essence of this proposal, which is based on the NCW pattern, is the transformation process. From the space missions of individual spacecraft (individual platforms) with various types of sensors and effectors on board, which perform functions related to planetary defense with a platform-centric approach, transformation to the system of many spacecraft connected in a network and integrated into network-centric systems with new cooperation algorithms based, is crucial.

As a result, it needs to obtain several network-centric capabilities, such as the following:

(1) New capabilities of the broadband space digital data transmission network with an appropriate quality of service, including reliability and security, guaranteeing the full interoperability of spacecraft and their subsystems: A Space INTERNET with a modern mobile protocol;

(2) Network capabilities of space sensor carriers of many intelligent classes, including autonomous sensors in space, forming the combined Space MEGA SENSOR of the future planetary defense system;

(3) Computational network capabilities of the spacecraft, which are nodes of the defense system, with supercomputing centers on board with mass-parallel computing architecture and performance of many tens of Teraflops for local data processing, information, and knowledge gathering for Space Situational Awareness (SSA) databases, forming a joint Space TERA GRID;

(4) Network capabilities of new kinetic and electromagnetic actuators for the precise destruction of small celestial bodies pose a threat to the Earth. Our space warheads will be able to change the trajectories of small celestial bodies on collision courses with the Earth, and they will serve as a network through connection with the Space MEGA SPACE-WARHEAD. A network of other systems is also possible, e.g., directed energy weapons based on Space MULTIDEW combined laser systems.

This list of potential capabilities suggests that the Future Planetary Defense System (FPDS) must be a Distributed Network-Centric System (see Figure 4). In this scheme, our nodes use the idea of Baran's work [18], which he named distributed communications. 


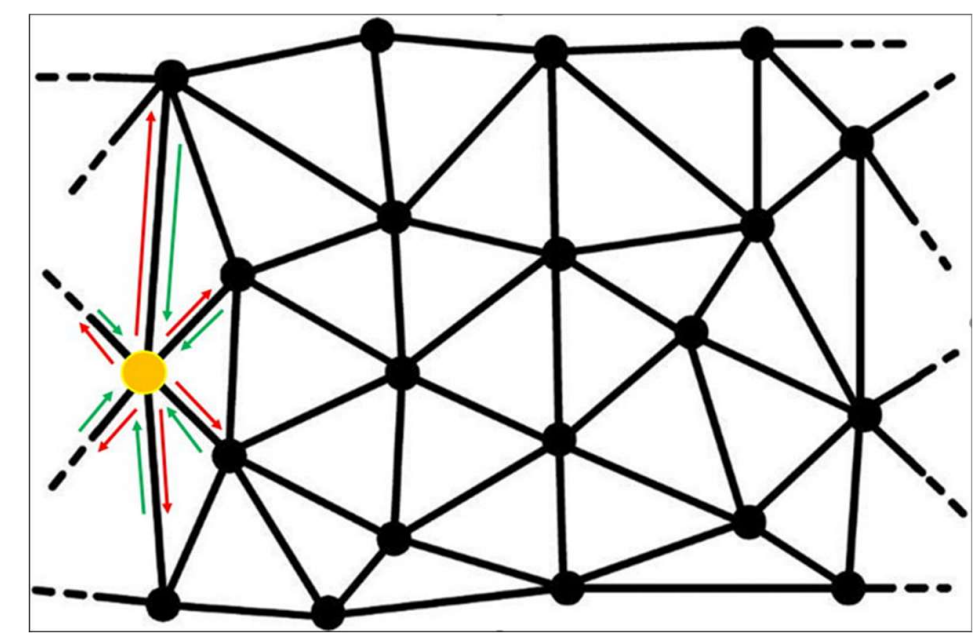

Figure 4. The projection of a 3D distributed network on a plane. The yellow node represents the primary spacecraft. The red arrow means the synchronization. The green arrow means the coordination.

A crucial part of FPDS Distributed Network Sensors - at the highest level of integration, being just a set of sensors on all spacecraft in the Solar System's inner space-is that it has high power nodes, with supercomputers on board, and with sophisticated computational applications. This primary FPDS subsystem will also create an extensive computing system with multi-TERAFLOPS of power in space. It will be a subsystem for processing useful informational data from sensors with the potential to model events in less time than those that occur in real-time; it will also serve to expand the Space Situational Awareness (SSA) of upcoming problems and support operators' knowledge during the commissioning and implementation of the planetary defense decision chain (see Figure $5)$.

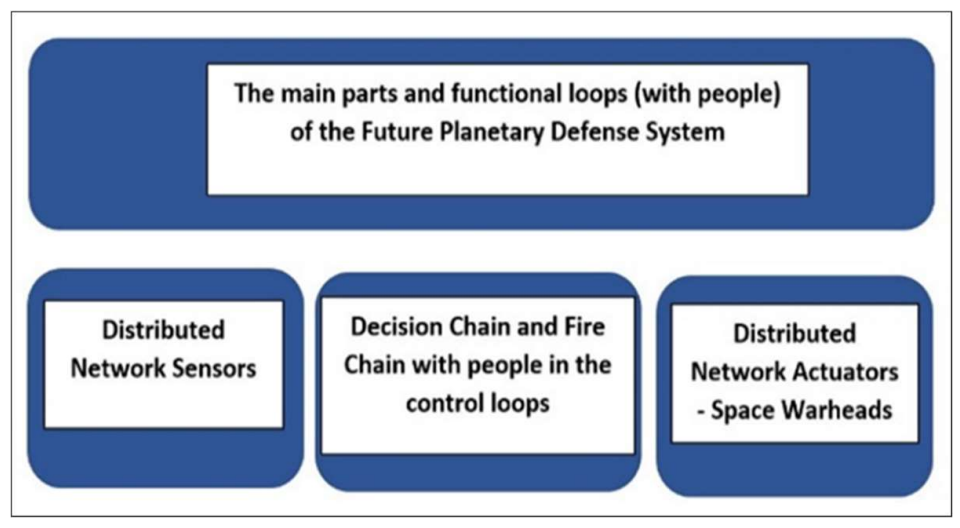

Figure 5. The main parts and functional loops of the Future Planetary Defense System - with people in the control loops.

The concept of autonomous sensor systems in spacecraft means that the classic definition of sensors as a transducer subsystem is insufficient. The top-down methodology of FPDS concept building requires the following modified Marian Mazur definition of an autonomous system with on-board sensors and actuators [10]: An autonomous system is a system with the capability to control itself and the ability to counteract the loss of its control capabilities.

Figure 6 shows the information and energy channels in autonomous spacecraft. 
For the subsystem node of the entire FPDS, i.e., an autonomous spacecraft with sensors and effectors, the definition can be supplemented with the following requirement. It should be a spacecraft capable of remaining in the environment for as long as possible.

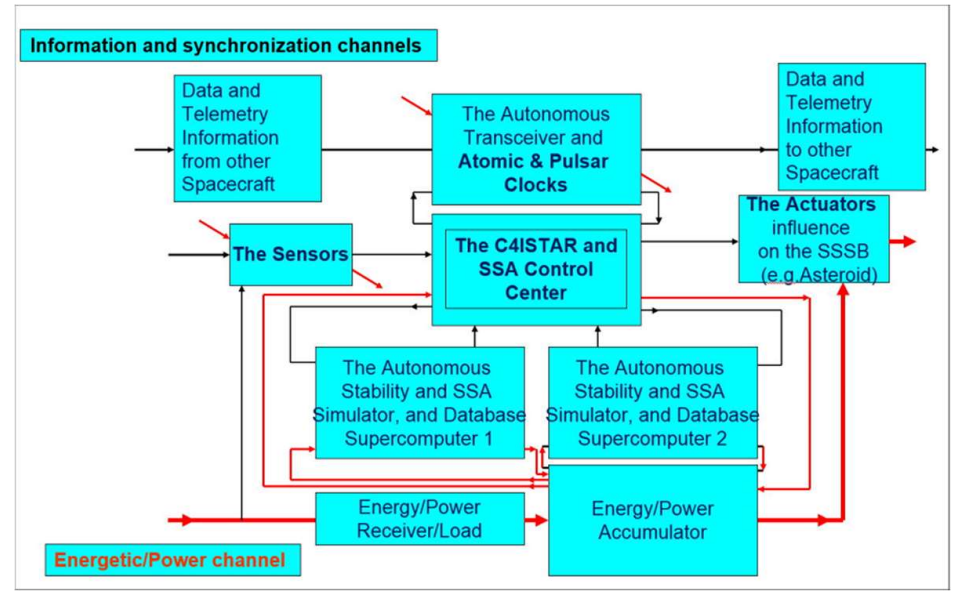

Figure 6. Shows here the modification and extension of Marian Mazur's scheme of an autonomous system with application to FPDS's spacecraft. SSSB is a Small Solar System body; SSA is Space Situational Awareness.

It means that the autonomous system must contain the relevant components, as subsystems, and above all, the following:

(1) intelligent sensors, i.e., components for collecting information from the environment;

(2) special sensors for system synchronization supported by atomic and pulsar clocks;

(3) C4ISTAR as local management and command center, authorized when needed by people, and collecting, processing, storing, and transferring information about the Space Situational Awareness (SSA); it must contain a homeostat and homeostasis stabilization subsystems, and duplicate supercomputers must support everything mentioned above;

(4) effectors, i.e., components to affect the environment.

Effectors should receive data locally from the sensors mentioned above. Globally, they should receive data, information, and commands authorized, if necessary, by people, from C4ISTAR, for which an information channel is needed. C4ISTAR decides and determines which of the possible interactions are to occur and allocates energy resources, enabling the work necessary for the effectors to perform the interaction; for this, an energy channel is needed that includes the following:

(5) technical systems for obtaining energy from the environment;

(6) local energy sources (energy and power generators);

(7) energy storage and conversion batteries;

(8) technical energy distribution and processing systems.

Since we are preparing a future system, we are interested in two categories of problems: cognitive, related to data collection (by flocks (!) of intelligent sensors), and the second is decisionmaking. For the requirements of the future planetary defense system, we can directly use the following slightly modified NCW Tenets associated with data collection and information processing [6]:

Tenet 1: A robustly networked spacecraft improves information sharing.

Tenet 2: Information sharing and collaboration enhance the quality of information and shared Space Situational Awareness (SSA).

Tenet 3: Shared Space Situational Awareness (SSA) enables self-synchronization.

Tenet 4: Self-synchronization, in turn, dramatically increases mission effectiveness.

This multi-stage process is shown in Figure 7. 


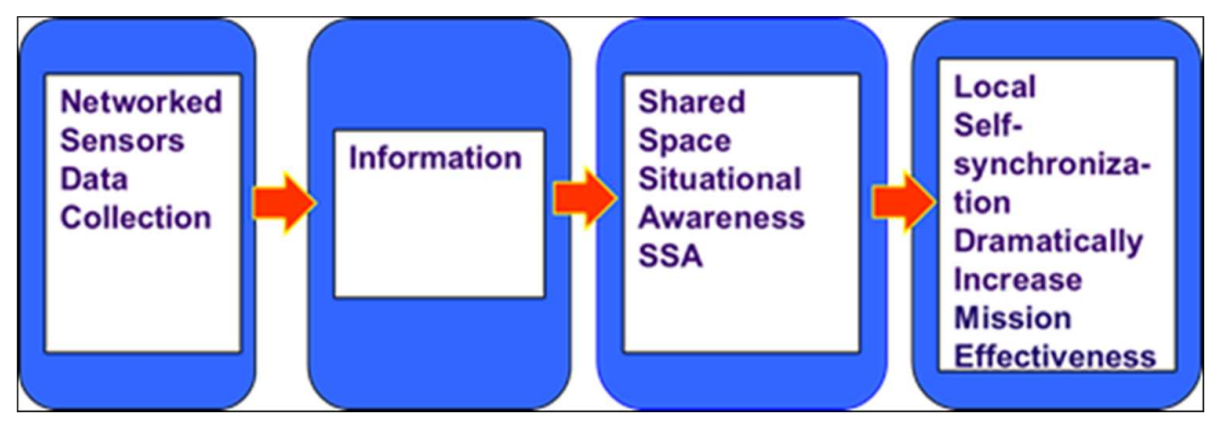

Figure 7. Slightly modified network-centric operations and warfare (NCW) Tenets.

As a decision-making model at the early stage of building our preliminary computer concept of the Future Planetary Defense System, we chose Boyd's Observe-Orient-Decide-Act (OODA) loop [20]. NCW and Command and Control (C2) systems architecture theory adopted OODA without any change.

The OODA loop is transparent and straightforward, so that it will be useful at a new computer concept stage. For military practitioners, the transition from platform-oriented systems to integrated and "simple" network-oriented systems is not enough. We need to move from a network-oriented approach to a knowledge-based approach to defense systems [19]. Figure 8 shows the new chain of the knowledge-based approach. It will be much easier to close the OODA loop on the top of the chain.

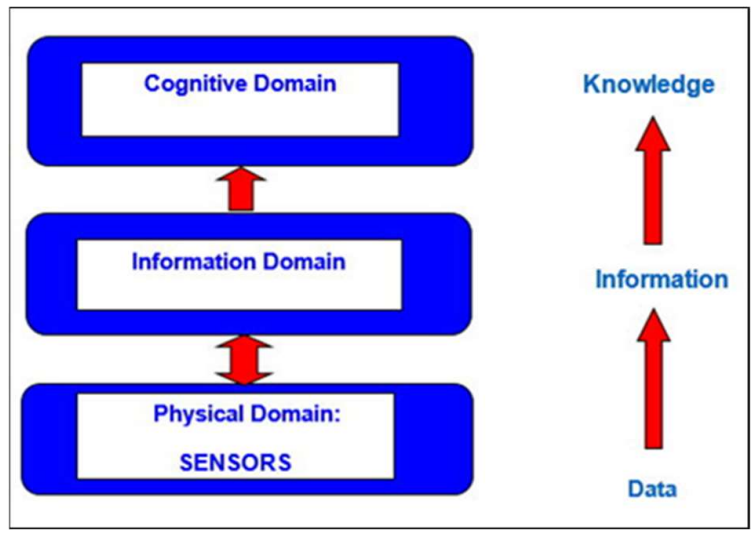

Figure 8. Knowledge-based approach.

In fact, in the FPDS concept, we will have at least three levels of hierarchy; the lower level is the hierarchy of autonomous sensors and actuators on spacecraft. The middle level is the hierarchy of autonomous spacecraft. The higher level is the hierarchy of FPDS spheres.

As the leading technologies for connecting nodes in the system, we propose laser and millimeterwave (MMW) technologies, but we will use the radio frequency (RF) technologies. By IAU definition, Small Solar System Bodies (SSSBs) are the Solar System objects that are neither planets, dwarf planets, nor natural satellites. Asteroids with diameters of $140 \mathrm{~m}, 100 \mathrm{~m}$, or slightly smaller, also being SSSBs, pose a high risk to the increasingly densely populated Earth's safety. Such bodies are already hazardous when they hit the surface of the Earth $[8,24]$.

The arsenal to combat threats from such celestial bodies, using energy in the range of 50-100 MT TNT, is already available using existing technology. It is possible to use this kind of actuator through contact or short-range kinetic interactions. We can apply classic explosives or nuclear warheads, and soon we may be able to use extremely high-powered lasers. But nuclear explosions can be a source of further threats to space vehicles, synchronization processes of space vehicles, and their electromagnetic compatibility (EMC). With repeated action, electromagnetic pulses of nuclear 
explosions in space may appear - from interactions with the plasma of the ionized matter of the bomb structure and with the plasma of the ionized remains of the asteroid. Another threat is a systemgenerated electromagnetic pulse (SGEMP) or spacecraft charging (SCC). However, it is not the end of the space mission; synchronized sensors are crucial in the next stage of the mission. After the attack, we will need a select category of sophisticated sensors to evaluate the attack results and confirm the success of the mission. To evaluate certain parameters, we will prepare a simple scenario and simulation of the deflection mission. For this reason, we must prepare a precise computer experiment to determine how our weapons will interact with the asteroid. We need to assess what deformations of the attacked asteroid we can expect and which sensors to choose to measure, from a safe distance, the level of damage.

\section{A concept of Future Planetary Defense System synchronization with an autonomous sensor hierarchy.}

The synchronization of the future Planetary Defense System (FPDS) will be difficult due to its environment and the extreme demands placed on the technologies used. The radius of action at astronomical distances found in the solar system and the complex tasks of combating small celestial bodies make the degree of difficulty above all what we have dealt with so far. Modeling synchronization processes is also difficult because it requires learning approximate methods of describing quantum phenomena and, on the other, approximate methods of describing relativistic phenomena. Autonomous quantum sensors such as atomic clocks and interferometers have just arrived onboard spacecraft in deep outer space and come from the microworld. Modeling and simulations of their internal operation are carried out using mechanics and quantum electrodynamics because they operate on a subatomic scale. Meanwhile, modeling the cooperation of these instruments within the planetary defense system's framework covers the largest known ranges on the cosmic scale. Everything will have to be rebuilt from scratch in this area, both the instruments and their models. Future technologies will have to be planned and pre-tested in computer models to new requirements. In turn, the presence of optical communication technology using photons, where quantum systems with laser transmitters and receivers of coherent detection (with homodyne and heterodyne) or direct detection (photon counting) are combined with the use of semi-classic models of shot-noise. Secure communications in space with the distribution of quantum cryptographic keys (QKD) is a future technology with applications in space. Remote sensing with classic measurements using electromagnetic waves and quantum (photon) instruments in the field of quantum metrology, e.g., laser radar systems, will be used in space for detection, parameter estimation, and imaging of small celestial bodies [21]. The entire class of autonomous sensors and actuators aboard the class of pico- and nanosatellites will be used for in situ measurements on small cosmic bodies' surface. We are talking about a set hierarchy of synchronization problems from the highest to the lowest level.

The highest level of the hierarchy concerns the synchronization of the entire FPDS, i.e., the coordination of events necessary for the proper interaction of all three system layers. The system's first layer is a sphere with a radius $r=1.25 \mathrm{AU}$ around the sun. The second layer of the system is a sphere with a radius $r=900,000 \mathrm{~km}$ around the Earth. We place the third layer of the system on the Earth's surface, covering the space around the Earth up to the height of the geostationary orbit. The essence of the problem is the synchronization of activities aimed at linking events into a cause-andeffect chain subject to the rules of decision making and issuing commands with people's participation in feedback loops. Synchronization processes must accompany the highest synchronization and event coordination at an intermediate level in this hierarchy. Synchronization signals must be exchanged between autonomous spacecraft, just like process synchronization signals between autonomous quantum sensors aboard these spacecraft. This basic level of the autonomous sensor hierarchy requires prior synchronization of the atomic clocks at an intermediate level of synchronization, i.e., onboard spacecraft. Pulsar clocks onboard the spacecraft, i.e., at an intermediate level of the hierarchy, will additionally support all synchronization processes due to their long-term stability. Ultimately, after overcoming these difficulties, the clocks of the individual autonomous sensors will be synchronized. But before that, these processes must be modeled and simulated in computers. 
Figure 9 is a snapshot of the interactive 3D visualization of Asteroid Space Asterank [22].

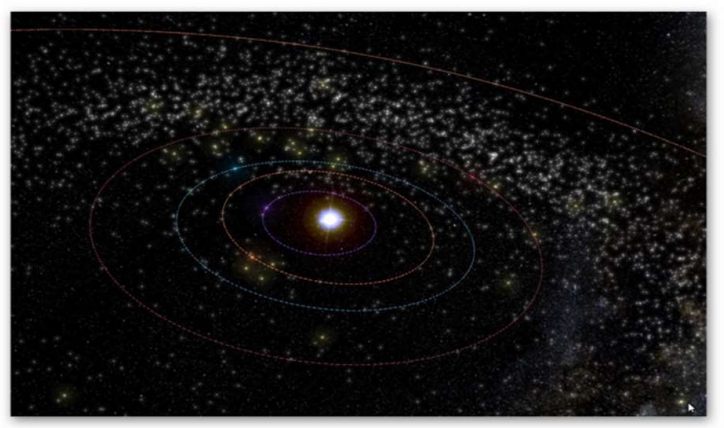

Figure 9. Snapshot from a 3D Interactive Asteroid Space Visualization-Asterank [22]. Details on orbits and basic physical parameters are from the Minor Planet Center and NASA JPL. In the centerthe Sun, Violet-Mercury orbit, Orange-Venus orbit, Blue-The Earth orbit, Red-Mars orbit, Brown-Jupiter orbit, Main Belt lies between Mars orbit and Jupiter orbit, in which white points are asteroids.

The modeling techniques used for these purposes are from various fields of science, e.g., Computational Physics and Astrophysics in Relativistic Frames of Special Relativity and General Relativity, and Computational Quantum Mechanics. We will model the clocks synchronization in the sense of the Poincare-Einstein convention, and FPDS synchronization events, as a coordination of the joint operation of the entire FPDS with self-synchronization effect of Space Situational Awareness (SSA) knowledge with people in the loop.

In Section 5, the simulation's results are given of the final part of asteroid deflection, which is a simple scenario against $100 \mathrm{~m}$ diameter asteroids. However, we first explain the relativistic model of motion.

\section{General relativity theory and Post-Newtonian Approximation (PN). Motion in PN Reference Systems within the framework of General Relativity}

The most fundamental theory of classical physics is General Relativity. Following the international recommendations of the IAU (International Astronomical Union), we will describe events occurring in space in the Solar System as well as near the Earth. Therefore, at the stage of the initial computerized concept of FPDS construction, as part of the preparation of a mathematicalphysical and numerical model for computer simulation of events occurring during the operation of the future planetary defense system, the theoretical description must be created within Einstein's General Relativity.

The Einstein field equations of the astrophysical applications inside the Solar System are:

$$
G_{\mu \nu}=\frac{8 \pi}{c^{4}} G T_{\mu \nu}
$$

where: $G_{\mu \nu}$ - is Einstein's tensor; $g_{\mu \nu}$ - is the metric tensor $(-,+,+,+)$; c - is the speed of light in a vacuum; $G$ - is the gravitational constant; and $T_{\mu \nu}$ - is the stress-energy tensor.

In General Relativity Theory we must solve the equations of motion and field equations simultaneously, which means, that the problem of motion cannot be strictly solved [1]. Only the approximate method can be used, which consists of the power series expansion of all functions appearing in the field equations against the negative powers of the speed of light. This problem was first solved in the work of Einstein, Infeld, and Hoffmann in 1938 [2].In 1960, Infeld and Plebański finished solving the problem of "Motion and Relativity" [3], in the spirit of Einstein, giving us, derived by the EIH approximation method, the relativistic n-body equations of motion. T.D. Moyer 
[25] used the Lagrangian function from the work of Infeld and Plebański to obtain the approximate relativistic equation of motion for determining the ephemeris in the Solar System. This is the procedure that led to the Newtonian equation of motion with Post-Newtonian corrections. By numerical integration, it is possible to prepare the ephemeris for SSSBs and spacecraft of the FPDS within the Solar System. The equation from Montenbruck and Gill [26] work is the same as that from the work of Moyer's [25]:

$$
\ddot{r}=-\frac{G M_{\odot}}{r^{2}}\left(\left(4 \frac{G M_{\odot}}{c^{2} r}-\frac{v^{2}}{c^{2}}\right) \cdot \vec{e}_{r}+4 \frac{v^{2}}{c^{2}}\left(\vec{e}_{r} \cdot \vec{e}_{v}\right) \vec{e}_{v}\right)
$$

Moyer in sixties of the last century prepared his work [25] based on Infeld work from Infeld and Plebański monography [3]. Spier [

$$
\ddot{\vec{r}}=-\mu_{c} \frac{\vec{r}}{r^{3}}+\sum_{i} \mu_{i}\left[\frac{\vec{r}_{i c}}{r_{i c}{ }^{3}}-\frac{\vec{r}_{i p}}{r_{i p}{ }^{3}}\right]+\ddot{\vec{r}}(O B L)+\ddot{\vec{r}}(S R P, A C)+\ddot{\vec{r}}(M B)+\ddot{\vec{r}}(I O B L)+\ddot{\vec{r}}(G R)
$$

where

$\ddot{\vec{r}}=$ acceleration of spacecraft

$\mu_{c}=$ gravitational constant of the center of integration, $\mathrm{km}^{3} / \mathrm{sec}$

$\mu_{i}=$ gravitational constant of body $\mathrm{i}, \mathrm{km}^{3} / \mathrm{s}^{2}$

$\vec{r}=$ position of spacecraft relative to center of integration

in 1950.0 earth equatorial rectangular coordinates

$\vec{r}_{i c}=$ position of body $i$ relative to center of integration

in 1950.0 rectangular coordinates

$\vec{r}_{i p}=$ position of spacecraft relative to body $\mathrm{i}$ in 1950.0

rectangular coordinates.

$\ddot{\vec{r}}_{i}(j)=\frac{\mu_{j}\left(\vec{r}_{j}-\vec{r}_{i}\right)}{r_{i j}{ }^{3}}\left\{-\frac{4}{c^{2}} \phi_{i}-\frac{1}{c^{2}} \phi_{j}+\left(\frac{\dot{s_{i}}}{c}\right)^{2}+2\left(\frac{\dot{s_{j}}}{c}\right)^{2}-\frac{4}{c^{2}} \dot{\vec{r}}_{i} \cdot \dot{\vec{r}}_{j}-\frac{3}{2 c^{2}}\left[\frac{\left(\vec{r}_{i}-\vec{r}_{j}\right) \cdot \dot{\vec{r}}_{j}}{r_{i j}}\right]^{2}+\frac{1}{2 c^{2}}\left(\vec{r}_{j}-\vec{r}_{i}\right) \cdot \ddot{\vec{r}}_{j}\right\}+$

$+\frac{1}{c^{2}} \frac{\mu_{j}}{r_{i j}^{3}}\left[\left(\vec{r}_{i}-\vec{r}_{j}\right) \cdot\left(4 \dot{\vec{r}}_{i}-3 \dot{\vec{r}}_{j}\right)\right]\left(\dot{\vec{r}}_{i}-\dot{\vec{r}}_{j}\right)+\frac{7}{2 c^{2}} \frac{\mu_{j} \ddot{\vec{r}}_{j}}{r_{i j}}$

$r_{i j}=$ coordinate distance between bodies $\mathrm{i}$ and $\mathrm{i}$

$\left(s_{i}\right)^{2},\left(s_{j}\right)^{2}=$ square of velocity of bodies $\mathrm{i}$ and $\mathrm{j}$, respectively

$\phi_{i}=$ Newtonian potential at body $\mathrm{i}$

$\phi_{i}=$ Newtonian potential at body $\mathrm{j}$

and

$\ddot{\vec{r}}_{i j}=\sum_{m \neq j} \frac{\mu_{m}\left(\vec{r}_{m}-\vec{r}_{j}\right)}{r_{m j}^{3}}$ 
For example a circular orbit $\frac{G M_{\odot}}{r}=v^{2}$, and a relativistic correction of acceleration, a simple equation is given:

$$
\ddot{r}=-\frac{G M_{\odot}}{r^{2}} \vec{e}_{r}\left(3 \frac{v^{2}}{c^{2}}\right)
$$

When the XXXth General Assembly IAU (2018) recommends a reference system in the framework of General Relativity in the Post-Newtonian approximation. It became an imperative for our concept, because any mathematical and physical computer modeling of the system is related to the selection of an appropriate reference system. Fortunately, for interactions of very small contact bodies, we can use classical Mechanics of Continuous Media.

\section{The threat from a $\mathbf{1 0 0} \mathrm{m}$ diameter asteroid impact on Earth estimation $[8,23]$.}

Thanks to Mark Boslough's work [8] analysis, the main risk to the Earth population, from Small Solar System Bodies (SSSBs), was estimated at the $100 \mathrm{~m}$ diameter body. To estimate with a mathematical and physical model a realistic threat to the Earth's population from a $100 \mathrm{~m}$ diameter asteroid impact, we used the ImpactEarth! Portal available in the network [23], and based on an article by G.S. Collins, H.J. Melosh, and R.A. Marcus, "Earth Impact Effects Program: A Web-based computer program for calculating the regional environmental consequences of a meteoroid impact on Earth" [24] giving approximate results of modeling, with a high level of confidence. Initial data, characteristics of the asteroid, the calculation results are provided below:

Initial data:

Diameter: $100 \mathrm{~m}$;

Density of rock: $2400 \mathrm{~kg} / \mathrm{m}^{3}$;

Angle of impact: 45 degrees;

Initial velocity: $20 \mathrm{~km} / \mathrm{s}$;

Target: Sedimentary rock;

Distance to the point of impact: $1 \mathrm{~km}$;

Mass: $1.0 \cdot 10^{10} \mathrm{~kg}$

Results of computations:

Energy before atmospheric entry: $2.51 \cdot 10^{17}$ Joules $=60.0$ MegaTons TNT;

Impact Frequency: The average interval between impacts of this size somewhere on Earth during the last 4 billion years is $4.9 \cdot 10{ }^{3}$ years.

Atmospheric Entry: The projectile begins to breakup at an altitude of 63300 meters. The projectile reaches the ground in a broken condition. The mass of projectile strikes the surface at velocity 4.45 $\mathrm{km} / \mathrm{s}$.

The impact energy is $1.24 \cdot 10^{16}$ Joules $=2.97$ MegaTons TNT;

The broken projectile fragments strike the ground in an ellipse of dimension $0.917 \mathrm{~km}$ by $0.648 \mathrm{~km}$. Crater Dimensions: Crater shape is normal in spite of atmospheric crushing; fragments are not significantly dispersed;

Transient Crater Diameter: 904 meters;

Transient Crater Depth: 320 meters;

Final Crater Diameter: $1.13 \mathrm{~km}$;

Final Crater Depth: 240 meters;

The crater formed is a simple crater;

The floor of the crater is underlain by a lens of broken rock debris (breccia) with a maximum thickness of 111 meters. At this impact velocity $(<12 \mathrm{~km} / \mathrm{s}$ ), little shock melting of the target occurs. Airblast: The air blast will arrive approximately 3.03 seconds after impact. 
Peak Overpressure: $1580000 \mathrm{~Pa}=15.8$ bars.

Max wind velocity: $976 \mathrm{~m} / \mathrm{s}$.

Sound Intensity: $124 \mathrm{~dB}$ (Dangerously Loud).

Damage Description: Multistory wall-bearing buildings will collapse.

Wood frame buildings will almost completely collapse.

Multistory steel-framed office-type buildings will suffer extreme frame distortion, incipient collapse.

Highway truss bridges will collapse.

Highway girder bridges will collapse.

Glass windows will shatter.

Cars and trucks will be largely displaced and grossly distorted and will require rebuilding before use.

Up to 90 percent of trees blown down; remainder stripped of branches and leaves.

The simulation results indicate that an area exceeding $300 \mathrm{~km}^{2}$ would be seriously damaged.

\section{The final part of the FPDS deflection mission modeling and simulations}

To verify the possibility of destruction, damage or redirection to another orbit of $100 \mathrm{~m}$ in diameter asteroids, using technology available on Earth, we decided to use our hydrocode, using the K. Jach "free particles" method $[13,14]$ to simulate physically faithful interactions on stone asteroids with a density of $2400 \mathrm{~kg} / \mathrm{m}^{3}$ and a diameter of $100 \mathrm{~m}$. We used three variants of events with targets within hundred meters order:

1) asteroid onto asteroid impact, 2) conventional explosive charge detonation deflection, and 3) hypothetical laser deflection in some simplified configurations.

We prepared the full mathematical-physical model [15] starting with a hydrostatic model of asteroid with $100 \mathrm{~m}$ in diameter. The $2.5 \mathrm{D}$ cylindrical, axially symmetric $(\mathrm{r}, \mathrm{z})$ coordinate system with a spherical asteroid was applied, as seen in Figure 8.

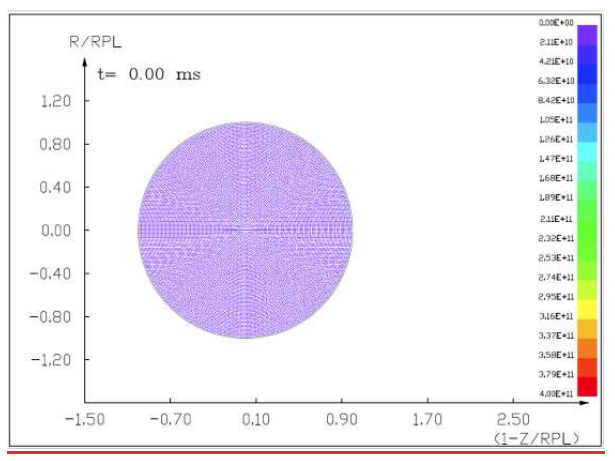

Figure 10. A homogeneous, computational mesh of the asteroid at the initial moment $(t=0.0 \mathrm{~s})$ shows the uniform pressures inside.

We did not include the strength of the material. The spherical symmetry of the undisturbed asteroid was assumed.

The initial density distribution $\mathrm{Q}(\mathrm{R})$ inside the asteroid is as follows:

$$
\rho=\rho_{0}(R), \quad 0<R<R_{0}
$$

where $\mathrm{R}_{0}$ denotes the asteroid radius at the initial moment of time. 
The initial distribution of Grüneisen parameter $\gamma$ inside the asteroid is as follows:

$$
\gamma=\gamma_{0}(R), \quad 0<R<R_{0}
$$

The Grüneisen parameter $\gamma$ is assumed to be undisturbed.

The equation of the state of the asteroid's material is consistent with the following form of the Mie-Grüneisen equation of state, in which the first part is similar to the pressure-density relationship of Murnaghan, and the second part is the Grüneisen term:

$$
p-p_{0}=\frac{K_{0}}{K_{0}^{\prime}}\left[\left(\frac{\rho}{\rho_{0}}\right)^{K_{0}^{\prime}}-1\right]+\gamma \rho\left(E-E_{0}\right)
$$

where $\mathrm{p}$ is the pressure, $\mathrm{Q}$ is the density, and $\mathrm{E}$ denotes the specific energy per unit of mass. Ko denotes the initial bulk modulus, which depends linearly on the undisturbed pressure $\mathrm{p}_{0}$ :

$$
K_{0} \text { is identical to } K\left(p_{0}\right)=K_{00}+K_{0}^{\prime} \cdot p_{0}
$$

For silicate rock:

$$
\left.\begin{array}{c}
K_{00}=1.5 \cdot 10^{11} P a \\
K_{0}^{\prime}=3.3
\end{array}\right\}
$$

The initial and undisturbed pressure $\mathrm{p}_{0}$ are determined by the hydrostatic equation:

$$
d p_{0}=-\frac{G m_{0}(R)}{R^{2}} \rho_{0}(R) d R
$$

where $G=6.6743 \cdot 10^{-11} \mathrm{~m}^{3} \cdot \mathrm{kg}^{-1} \mathrm{~s}^{-2}$ is the gravity constant and $\mathrm{m}_{0}(\mathrm{R})$ is the partial mass within an undisturbed sphere of radius $\mathrm{R}$.

$$
m_{0}=4 \pi \int_{0}^{R} \rho_{0}(x) x^{2} d x
$$

The density of the impactor is assumed to be equal to $2400 \mathrm{~kg} / \mathrm{m} 3$.

Construction of a hydrodynamic mathematical-physical model of the asteroid deflection process from the collision course began with the preparation of an appropriate system of partial differential equations that describe the relevant conservation laws. The numerical model is a hydrocode of the free particle method (HEFPM) [16,14].

The following set of equations of mass, momentum and energy conservation was used:

Mass:

$$
\frac{d \rho}{d t}+\rho\left(\frac{\partial u}{\partial r}+\frac{\partial v}{\partial z}+\frac{u}{r}\right)=0
$$

Momentum:

$$
\begin{gathered}
\rho \frac{d u}{d t}=-\frac{G m_{0} \rho}{R^{2}} \frac{r}{R}-\frac{\partial p}{\partial r} \\
\rho \frac{d v}{d t}=-\frac{G m_{0} \rho}{R^{2}} \frac{z}{R}-\frac{\partial p}{\partial z}
\end{gathered}
$$


Energy:

$$
\rho \frac{d E}{d t}=-p\left(\frac{\partial u}{\partial r}+\frac{\partial v}{\partial z}+\frac{u}{r}\right)
$$

where

$$
\frac{d}{d t}=\frac{\partial}{\partial t}+u \frac{\partial}{\partial r}+v \frac{\partial}{\partial z}
$$

The boundary condition (pressure) on the free surface of the asteroid is equal to zero:

$$
p\left(R_{A s}\right)=0
$$

The equations of this problem can only be solved by numerical means. Nine consecutive figures (Figures 9-17) show the visualization of the hydrocode simulation results, as the time evolution of asteroid response on asteroid onto asteroid impact, the next as the conventional explosive charge detonation deflection, and the last as the hypothetical laser deflection. The colors are related to the pressure scale.

6.1) The results of the computer simulation of $100 \mathrm{~m}$ diameter asteroid deflection by impact of another asteroid $13.5 \mathrm{~m}$ in diameter with a velocity of $10 \mathrm{~km} / \mathrm{s}$ and $3 \mathrm{~km} / \mathrm{s}$ :

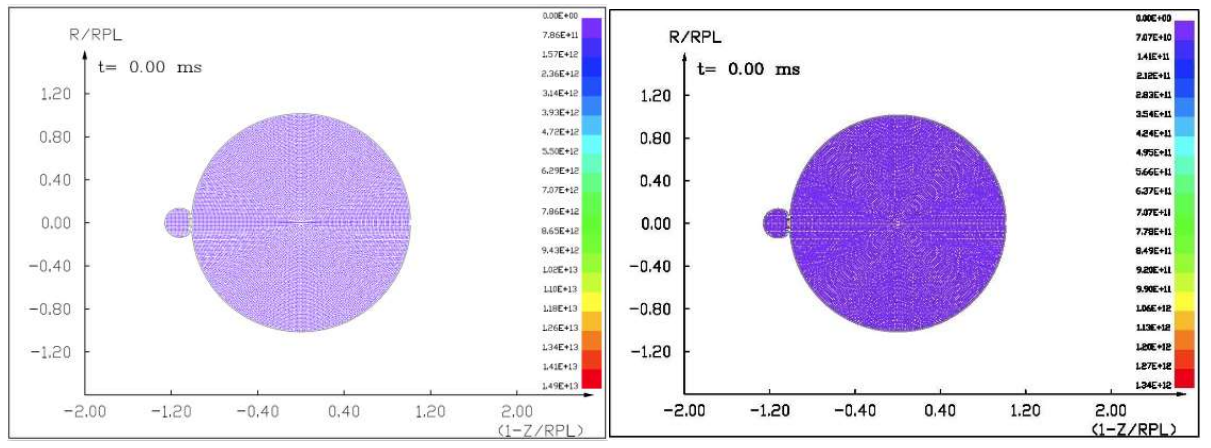

Figure 11. The initial stage of the computer simulation of $100 \mathrm{~m}$ diameter asteroid deflection using a smaller $13.5 \mathrm{~m}$ diameter asteroid. Initial velocity of smaller asteroid is $10 \mathrm{~km} / \mathrm{s}$ and $3 \mathrm{~km} / \mathrm{s}$

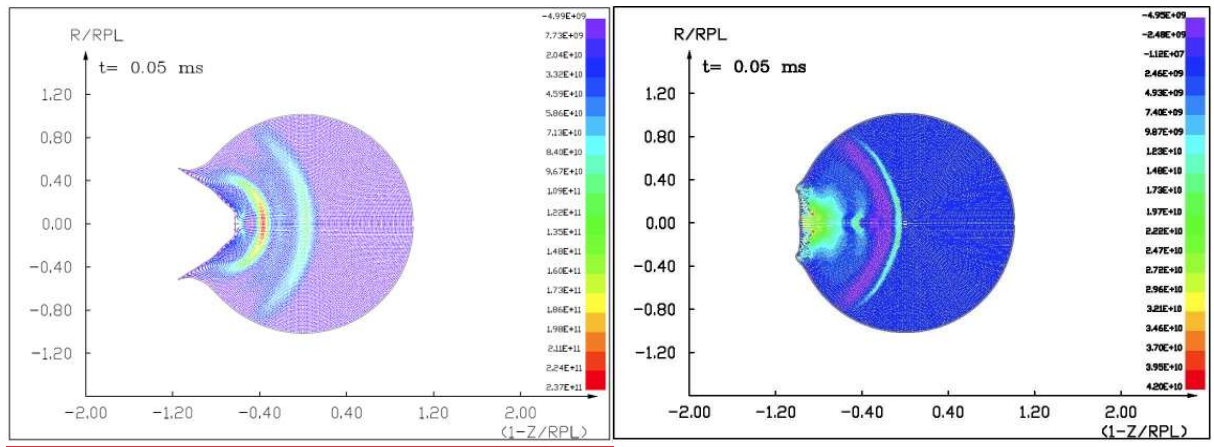

Figure 12. The next step in the visualization of the computer simulation of $100 \mathrm{~m}$ diameter asteroid deflection using a smaller $13.5 \mathrm{~m}$ diameter asteroid. We see a specific sequence of two shock waves in $5 \mathrm{~ms}$ after impact. 


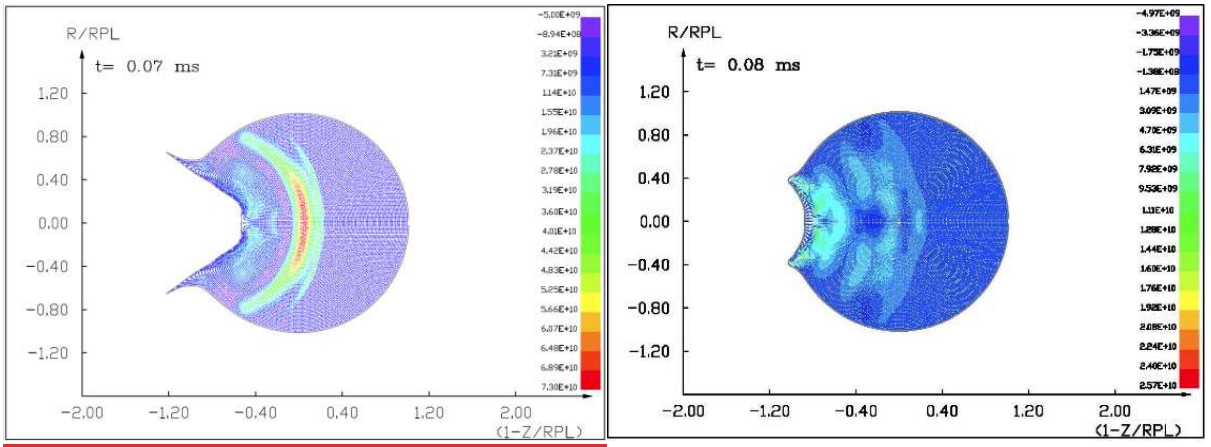

Figure 13. It is the next example of the computer simulation of $100 \mathrm{~m}$ diameter asteroid deflection using a smaller $13.5 \mathrm{~m}$ diameter asteroid. We see both shock waves moving together through a more massive asteroid in $7 \mathrm{~ms}$ after impact with velocity $10 \mathrm{~km} / \mathrm{s}$. In the next figure in $8 \mathrm{~ms}$ after impact with velocity $3 \mathrm{~km} / \mathrm{s}$ we see complicated structure of interacting shock waves.

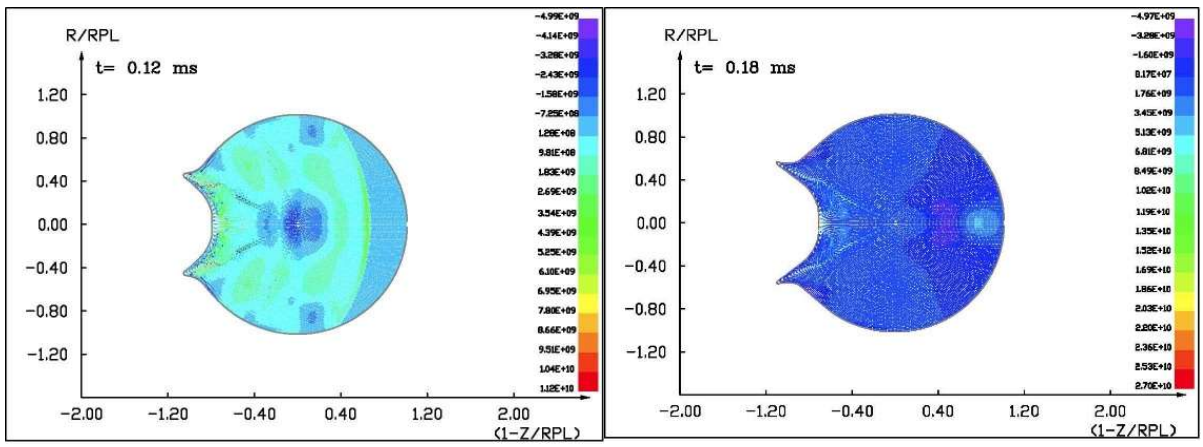

Figure 14. It is the next example of the computer simulation of $100 \mathrm{~m}$ diameter asteroid deflection using a smaller $13.5 \mathrm{~m}$ diameter asteroid. In the next figure in 12 and $18 \mathrm{~ms}$ after impact with velocity $3 \mathrm{~km} / \mathrm{s}$ we see complicated structure of interacting shock waves and cratering process.

6.2) The results of the computer simulation of an asteroid deflection using a conventional explosive with an octagen charge mass of $7100 \mathrm{~kg}$ :
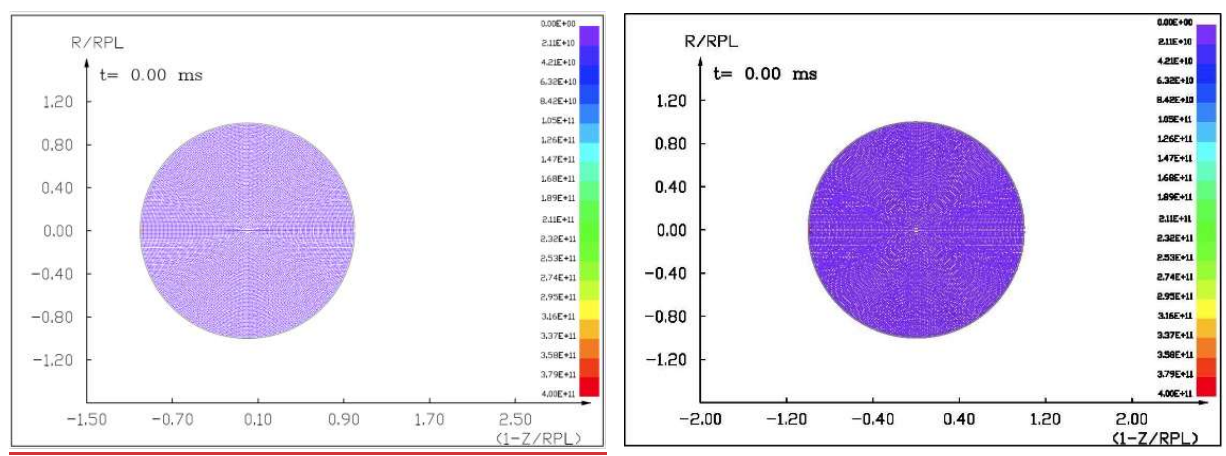

Figure 15. The initial stage of the computer simulation of $100 \mathrm{~m}$ diameter asteroid deflection using conventional explosives. Initial conditions, $\mathrm{t}=0, \mathrm{p}_{0}=401 \mathrm{~kb}$. Octagen charge mass equals $7100 \mathrm{~kg}$. 

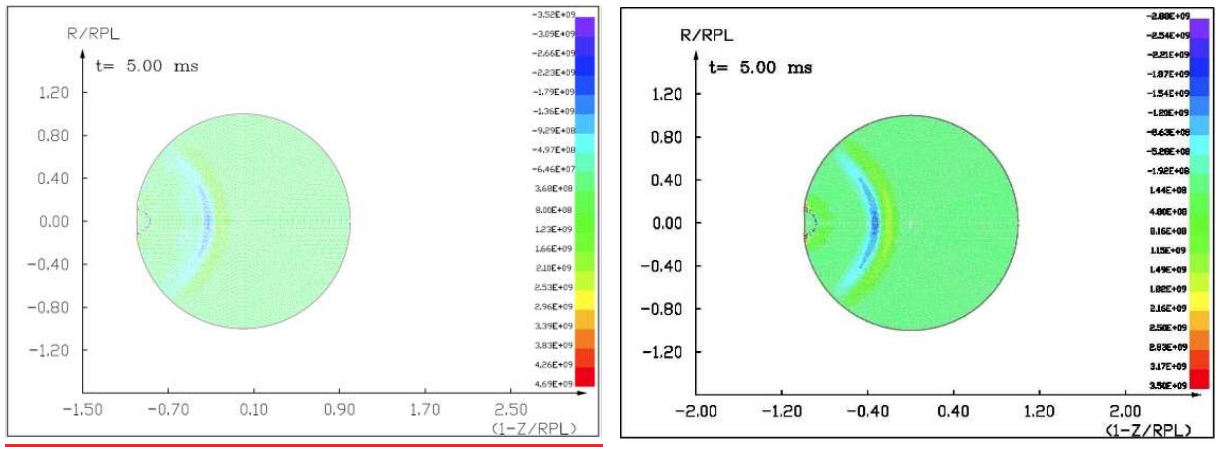

Figure 16. The next stage visualization of the computer simulation of $100 \mathrm{~m}$ diameter asteroid deflection using conventional explosives. We see crater and front of shock in $5 \mathrm{~ms}$ after detonation.
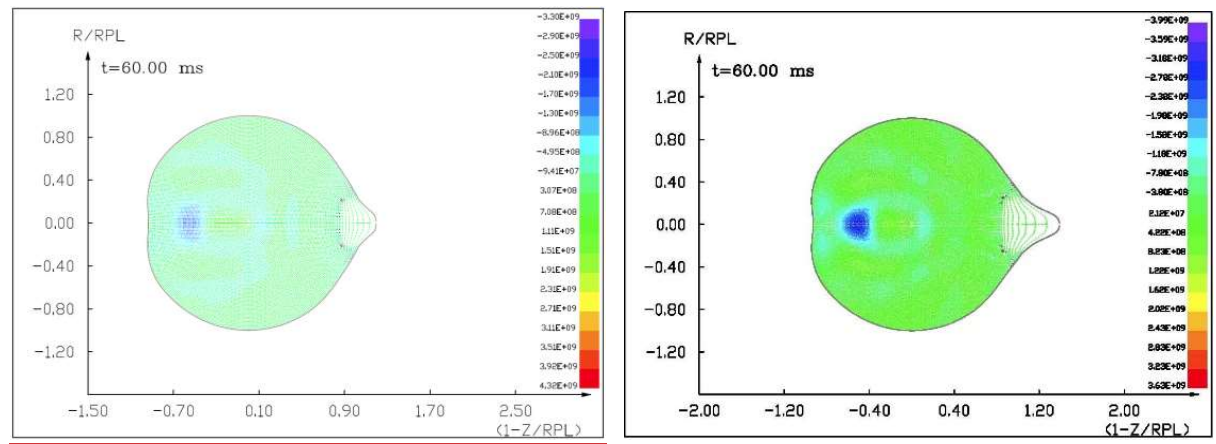

Figure 17. It is the last visualization of the computer simulation of $100 \mathrm{~m}$ diameter asteroid deflection using conventional explosives. We see the fragmented interior of a more massive asteroid together with the spalling phenomenon of a fragment around the antipodal point in $60 \mathrm{~ms}$ after detonation

\section{3) The results of the computer simulation of $100 \mathrm{~m}$ diameter asteroid deflection using giant} laser pulse:
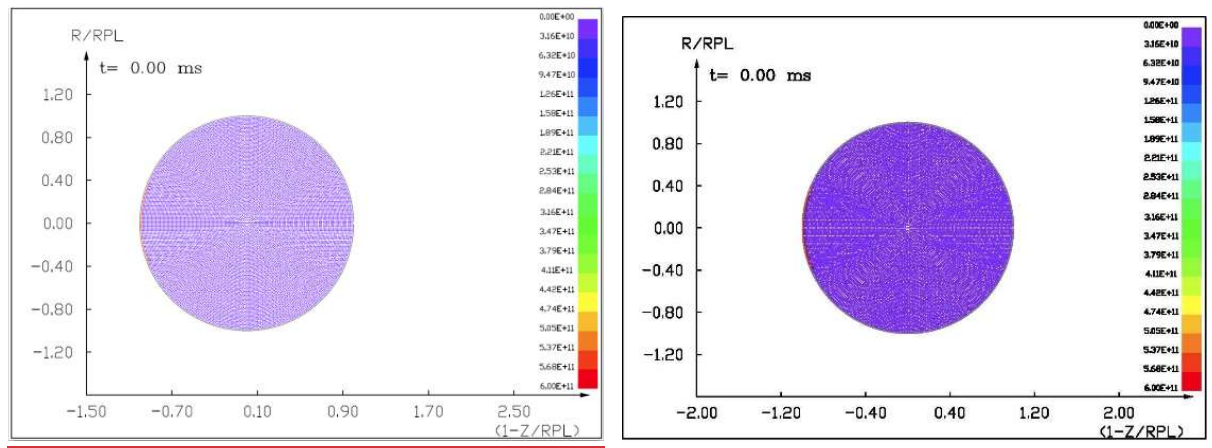

Figure 15. The initial stage of the computer simulation of $100 \mathrm{~m}$ diameter asteroid deflection using giant laser pulse. Initial conditions, $\mathrm{t}=0, \mathrm{p}_{0}=600 \mathrm{~kb}$. The radius of the ablation area is $20 \mathrm{~m}$. 

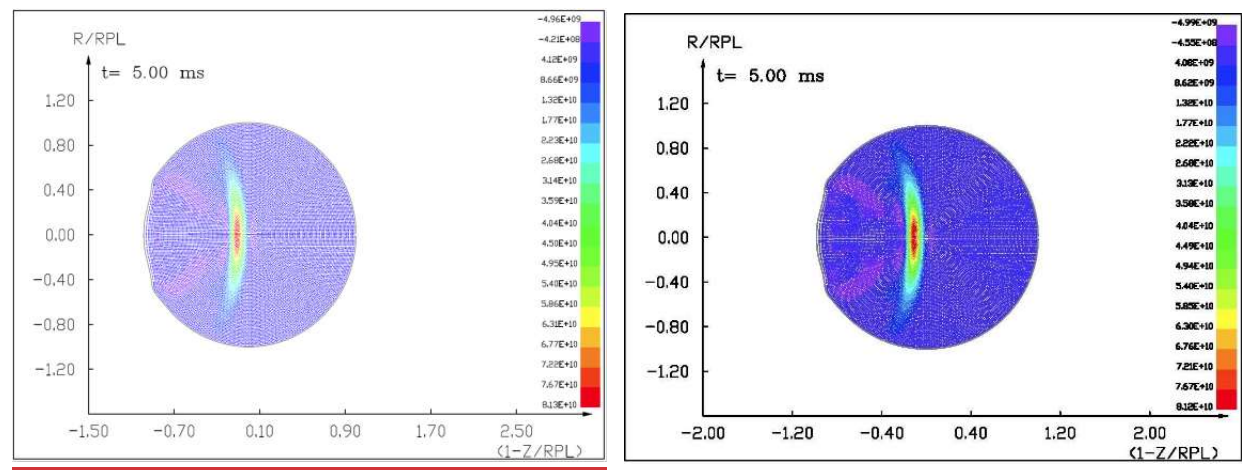

Figure 16. The next stage of the computer simulation of $100 \mathrm{~m}$ diameter asteroid deflection using giant laser pulse. We see the strong shock wave propagation in $5 \mathrm{~ms}$ after ablation.
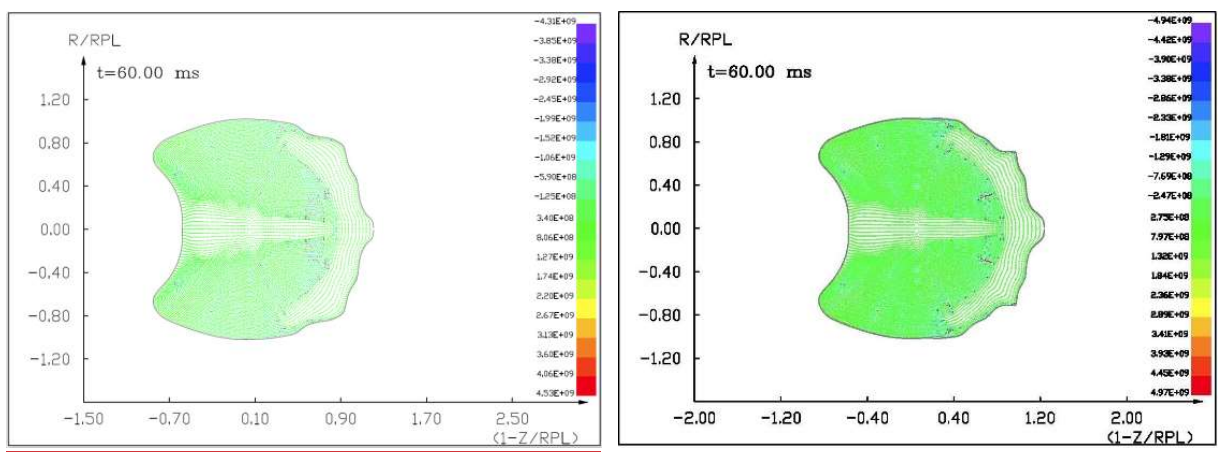

Figure 17. It is the last visualization of the computer simulation of $100 \mathrm{~m}$ diameter asteroid deflection using giant laser pulse. We see the large deformation of asteroid and completely fragmented interior together with the large spalling phenomenon of a great fragment around the antipodal point in $60 \mathrm{~ms}$ after ablation.

\section{Isotropic distribution of sensors on the ring's planes}

We focus on the outer spacecraft circular ring with a radius of $1.25 \mathrm{AU}$. In the first approximation, on each plane of the ring, we get the isotropic spacecraft distribution, and by using the appropriate autonomous spacecraft we have got the proper number of degree of arc e.g., with the initial number of spacecraft for modeling from 24 - every of 15 degrees of arc, through 36 spacecraft - every of 10 degrees of arc, through 72 spacecraft - every 5 degrees of arc.

In General Relativity (GR), the gravitational field affects and determines the metric laws of space-time [30]. For measurements, we will need clocks in inertial coordinate systems and "practically rigid bars" as in Special Relativity (SR). Each spacecraft will have a set of 3 to 4 atomic clocks for use in deep outer space.

Our concept of Future Planetary Defense System includes detection and continuous tracking of threats from space of the Solar System (from the Main Asteroid Belt and especially from the NearEarth Objects (NEOs)) together with combat strategy based on The Network-Centric Operation and Warfare theory with the flock algorithms [6].

In the inner part of the Solar System, at a distance $r=1.25 \mathrm{AU}$, we have to create the four rings of autonomous small spacecraft equipped with warheads. The spacecraft can operate in the four planes with common equinox vector. For the initial orbits calculations, and visualizations we used 
Asterank Portal prepared by Ian Webster [22]. Four planes deviate from the fundamental ecliptic plane: the first plane by ecliptic latitude is $b=0^{0}$ (Ring of Spacecraft b0), the second plane by ecliptic latitude is $b=45^{\circ}$ (Ring of Spacecraft b45), the third plane by ecliptic latitude is $b=90^{\circ}$ (Ring of Spacecraft b90), and the fourth plane by ecliptic latitude is $b=-45^{0}$ (Ring of Spacecraft b-45) as seen in Figures 18-21 [22].

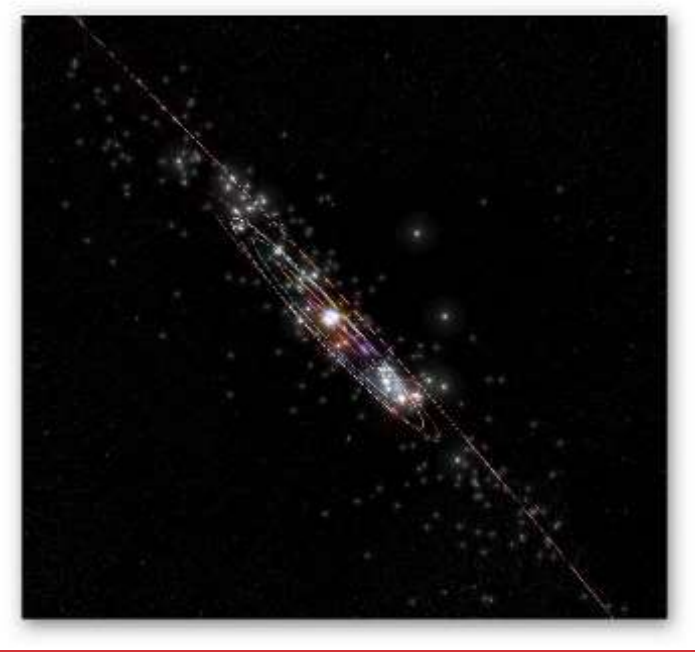

Figure 18. Ring of Spacecraft b0, with the ecliptic latitude $b=0^{0}$ [22].

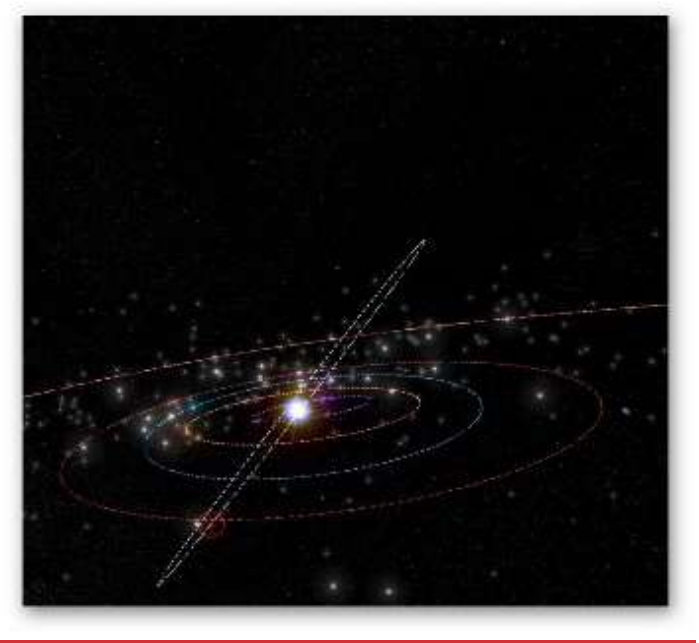

Figure 19. Ring of Spacecraft $b 45$, with the ecliptic latitude $b=45^{0}$ [22]. 


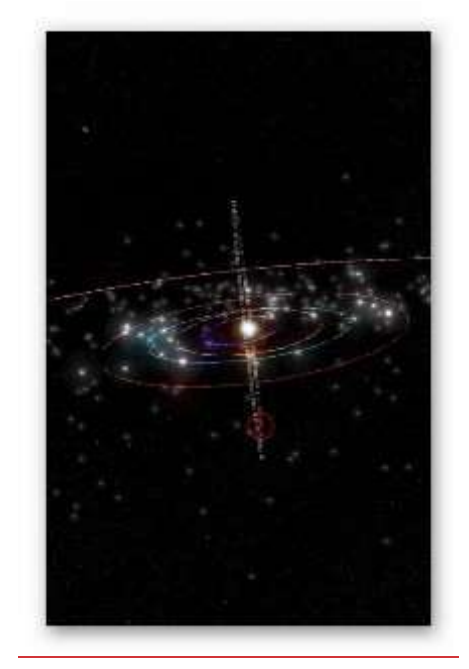

Figure 20. Ring of Spacecraft b90, with the ecliptic latitude $b=90^{\circ}$ [22].

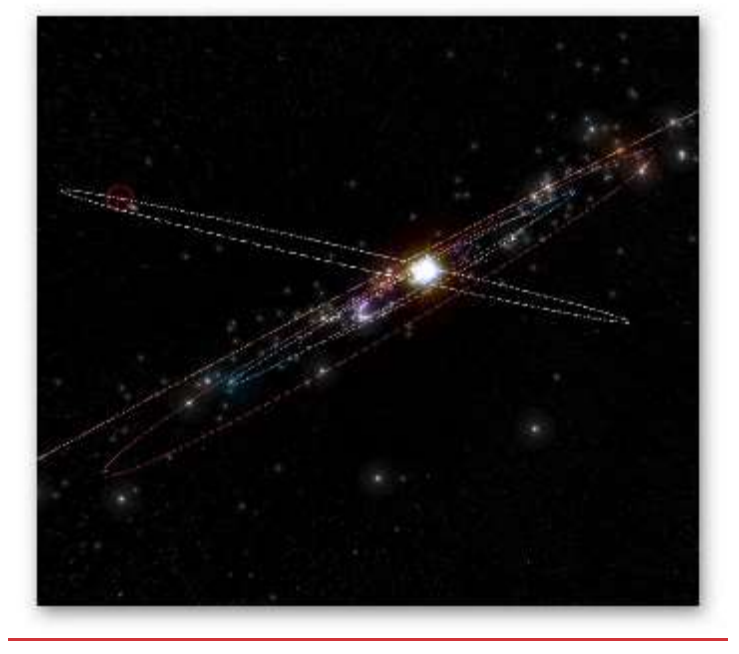

Figure 21. Ring of Spacecraft b-45, with the ecliptic latitude $b=-45^{0}$ [22].

\section{A few preliminary comments on the modeling of sensor synchronization in the FPDS}

1. High resolution and high accuracy measuring tools and methods come from quantum metrology research [27].

2. We focus on the outer circular ring with a radius of $1.25 \mathrm{AU}$ with the initial number of spacecraft for modeling ranging from 24 to 72 .

3. Each spacecraft will contain a set of 3 to 4 atomic clocks for use in deep space.

4. Each spacecraft will have a pulsar clock system.

5. There will be a laser transceiver system on every spacecraft.

6. Measurement, synchronization, and coordination will be based on the exchange of laser light pulses along the ring, on both sides, from the primary spacecraft. 
7. In General Relativity (GR), the gravitational field acts and determines the metric laws of spacetime [30]. For measurements and synchronization, we will need clocks in inertial coordinate systems and "practically rigid bars," as in Special Relativity (SR) [28,29].

8. Our task is to analyze, by computer simulations, the values of the gravitational field in the neighborhood of the FPDS space vehicle ring and the study of laser pulse propagation phenomena to meet STW conditions with high accuracy (the velocities of massive bodies in Solar System are very low compared to the speed of light).

\section{Summary}

The Planetary Defense System's success depends on the proper autonomous sensor system at the Space Metrology precision level. This System of Systems must work in Relative and Special theories Frameworks. The spherical navigation and precise positioning in General Relativity's framework need superior clocks because our sensors must find navigation positions by estimating the distance from three known fixed points measured by, for example, the flight time of a laser pulse. We need incredibly precise clocks for these purposes with atomic, Radio Pulsar, and X-ray Pulsar time. We need excellent clocks for system synchronization, coordination and correlation, perfect reference time scales, and perfect clock evaluation algorithms. We illustrated the concept and presented some key recommendations for such a relativistic metrology subsystem.

In this work, we introduced:

(1) relativistic mathematical-physical and numerical models of motion dynamics in relativistic coordinate frames in the Solar System;

and (2) quantum metrological models (functioning in systems described by relativistic motion models) for new types of quantum sensors. Examples of such autonomous sensors are atomic clocks for space vehicles in deep space (the prototype has been tested on a space vehicle since 2019).

(3) radio frequency pulsar clocks.

Other examples are sensors of space optical, millimeter, and radio data transmission systems.

The examples of autonomous sensors listed here require precise synchronization, coordination, and correlation.

New ideas and new proposals for computer models and programs must appear in this field of space techniques due to two groundbreaking changes made last year.

The first change is the obligation to implement the relevant resolutions of the 30th General Assembly of the IAU (International Astronomical Union) from January 1, 2019, regarding the use of relativistic reference systems.

The second groundbreaking change concerns the SI system since it was introduced on September 1, 2019, the so-called "The New SI System of Units" or "SI of 2018", which introduced quantum metrological standards.

New functional models of computer space mission simulators will now apply for autonomous spacecraft and their autonomous sensors. On a macro-scale, they are compatible with new mathematical and physical models based on general relativity. On a micro-scale, they will be compatible with models based on quantum mechanics and quantum electrodynamics.

The defense system proposal is entirely new and is based on the latest achievements and "knowhow" of the technology of large military network-oriented (network-centric) systems. It is a multilayered model of the future planetary defense system.

Until now, there were many limited proposals, for example, only directed against the so-called NEO: "An Architecture For Mitigating Near-Earth Object's Impact To The Earth" (978-1-5090-1613-6 / 17/3100 \$ @ 2017 IEEE). Most of the defense activities were analyzed near the ecliptic plane and with a single reaction against one asteroid.

Meanwhile, asteroids and comets that, because of collisions in the Main Belt of asteroids, may go on collision courses with Earth, those that will be several at once, and those that do not come from the Solar System, such as Oumuamua or Borisov-2, may pose a multiple threat. 
Everything we mention above requires new advanced programs (Computing of Big Data Management, Simulation, and Analytics).

New programs for computer simulation of future large planetary defense systems must be based on models that accurately reproduce

the physics of phenomena at the level of quantum metrology precision.

We are trying to improve the descriptions in the text because we describe new models modified by us to planetary defense.

It is our contribution. Our favor should be the results of an analysis of anti-plagiarism programs, which say that the work looks completely original.

We believe that the concerns of the reviewers will be clarified. We will present the new problems in applications of autonomous sensors distributed in Deep Space to a wide range of sensor specialists.

We try to demonstrate the configuration of an autonomous space vehicle for recognition, tracking, and possible destruction of asteroids.

The verification of synchronization methods and the selection of geometrical parameters of the system will belong to the later and more advanced parts of the work.

At the stage of the preliminary computer concept of the space mission (following the NASA, ESA, and the US DoD), it is too early to verify the synchronization methodology. Many detailed results of even preliminary computer simulations are still missing.

It is slightly different in our results of computer simulations of collisions and other effects on asteroids. They are our advantage, and they are computation.

We have got accurate results within the accepted models. According to these simulations, it will be possible to assess, e.g., the possibility of using selected sensors to analyze the impact of planned impacts on asteroids or comets.

The level of accuracy of simulations using our hydrocodes checked, for example, for the time of shock wave propagation to the Earth's antipodal point, is better than one promille.

When preparing the initial concept of planetary defense, the authors tried to clear up a few misconceptions and consider many factors resulting from the theory, knowledge, and military experience.

Some achievements, e.g., in the field of detection, recognition, and tracking, and command and control of ballistic missile systems, are inspiring and indicate many key success factors. All this should be done to avoid elementary mistakes in dealing with a potential threat.

Earlier analyzes show the high risk associated with the scale and scope of the destructive effects of an impact on a populated area of the Earth, even by a "tiny" asteroid $100 \mathrm{~m}$ in diameter.

Modern technologies applied in favorable conditions allow fighting asteroids with diameters up to $100 \mathrm{~m}$. Solutions to fighting larger celestial bodies by repeated use of classic attacks leading to the gradual destruction of asteroids and comets will be presented in subsequent articles. The problems of using nuclear charges for the same purposes require separate treatment.

\section{Acknowledgments}

This publication makes use of data products from the Near-Earth Object Wide-field Infrared Survey Explorer (NEOWISE), which is a project of the Jet Propulsion Laboratory/California Institute of Technology. NEOWISE is funded by the National Aeronautics and Space Administration.

\section{Conflicts of Interest:}

The authors declare no conflict of interest.

\section{References}

1. K. Jach, M. Mroczkowski, R. Świerczyński, E. Włodarczyk, P. Wolański," New numerical method for mechanics of continuous media", Computer Assisted Mechanics and Engineering Sciences (CAMES), 2: 105-128, 1995 
2. M. Mroczkowski, S. Kachel, A. Kozakiewicz," Space Metrology Problems of the Future Planetary Defense System with Pulsar Time, Navigation and Positioning", Conference Paper, IEEE Publisher: 2019 IEEE 5th International Workshop on Metrology for AeroSpace (MetroAeroSpace), Torino, Italy, 19-21 June 2019

3. M. Mroczkowski, S. Kachel, A. Kozakiewicz," Some Synchronization Problems of the Future Planetary Defense System's Autonomous Sensors Using Relativistic Space Metrology Tools", Unpublished

4. Einstein, A., Infeld, L., Hoffmann, B., (1938). "The Gravitational Equations and the Problem of Motion". Annals of Mathematics. Second series. 39 (1): 65-100

5. Infeld, L, Plebański, J. Motion and Relativity. Pergamon Press: Oxford, London, New York, Paris \& Państwowe Wydawnictwo Naukowe: Warszawa 1960

6. Network-centric warfare - Wikipedia. https://en.wikipedia.org/wiki/Network-centric_warfare

7. General Mission Analysis Tool (GMAT), https://software.nasa.gov/software/GSC-17177-1

8. M. Boslough, Airburst warning and response, Acta Astronautica, Vol. 103, October-November 2014, Pages 370-375

9. National Academies of Sciences, Engineering, and Medicine. 2019. Finding Hazardous Asteroids Using Infrared and Visible Wavelength Telescopes. Washington, DC: The National Academies Press. https://doi.org/10.17226/25476

10. M. Mazur Cybernetic theory of autonomous systems (in Polish). PWN, Warsaw 1966; http://www.autonom.edu.pl; Cybernetyczna teoria układów samodzielnych. PWN, Warszawa 1966

11. Department of Defense Architecture Framework (DoDAF)

https://en.wikipedia.org/wiki/Department_of_Defense_Architecture_Framework

12. https://en.wikipedia.org/wiki/\%CA\%BBOumuamua

13. https://en.wikipedia.org/wiki/2I/Borisov

14. Wide-field Infrared Survey Explorer - Wikipedia, https://en.wikipedia.org/wiki/Wide-field_Infrared_Survey_Explorer\#NEOWISE_(pre-hibernation)

15. NEOWISE: Surface Properties of 100+ Asteroids - Starship, https://asterisk.apod.com/viewtopic.php?t=38341\&p=282998

16. NEOWISE 2019 Data Release, http://wise2.ipac.caltech.edu/docs/release/neowise/

17. Jach K., Modelowanie komputerowe zjawisk kumulacyjnych, WAT, Warszawa, 1990. (Computer modelling of cumulation phenomena)(in Polish)

18. K. Jach, M. Mroczkowski, P. Wolański, Czislennoje modielirowanije procesow stolknoweni bolszich asteroidow s Zemlej (Numeryczne modelowanie procesów zderzeń wielkich asteroidów z Ziemią), I-e Wsechsojuznoje Sowieszczanije po temie "Asteroidnaja opasnost”, 10-11.1991, Proc. of All-Union Conference (with international participation) „Asteroid Hazard”, Institute of Theoretical Astronomy of Russian Academy of Sciences, Sankt Petersburg, 1992.

19. K. Jach, M. Mroczkowski, R. Świerczyński, P. Wolański, Dynamical Processes Associated with Collisions of a Medium Size Asteroid with the Primordial Earth, Bull. Am. Astron. Soc., 24, p.953, 1992.

20. K. Jach, M. Mroczkowski, R. Świerczyński, P. Wolański, Numerical calculations of dynamical effects of high velocity collisions (Obliczenia numeryczne dynamicznych efektów zderzeń ciał z dużymi prędkościami), Proceedings of the First Japanese-Polish Joint Seminar on Advanced Computer Simulation, Tokyo, November, pp. 39-51, 1993.

21. Mroczkowski M. Analiza teoretyczna dynamicznych efektów zderzenia dużych asteroid z ciałami planetarnymi, Rozprawa doktorska, Politechnika Warszawska, Warszawa,1994. (Theoretical analysis of the dynamic effects of the collision of large asteroids with planetary bodies, Dissertation) (in Polish)

22. K. Jach, J. Leliwa-Kopystynski, M. Mroczkowski, R. Swierczynski and P. Wolanski, Free particle modelling of hypervelocity asteroid collisions with the Earth, Planet. Space Sci., Vol. 42, No. 12, pp. 1123 1137,1994

23. G.Q. Chen, T.J. Ahrens, "Erosion of terrestrial planet atmosphere by surface motion after a large impact", Physics of the Earth and Planetary Interiors, 100 (1997): 21-26

24. Jach K, A. Morka, M. Mroczkowski, R. Panowicz, A. Sarzyński, W. Stępniewski, R. Świerczynski, J. Tyl, Komputerowe modelowanie dynamicznych oddziaływań ciał metodą punktów swobodnych, Wydawnictwo Naukowe PWN, Warszawa, 2001. (Computer modelling of dynamic interaction of bodies using free particles method) (in Polish) 
25. N. Mastrodemos, D.G. Kubitschek and S.P. Synnott, Autonomous navigation for the Deep Impact mission encounter with Comet Tempel 1, Space Science Reviews (2005) 117: 95-121.

26. P. Baran, RAND/RM-3420, 1964.

27. T. Hobbins, Cultural Shift, C4ISR The Journal of Net-Centric Warfare, vol.6, no.7, August 2007.

28. OODA https://en.wikipedia.org/wiki/OODA_loop

29. B.I. Erkmen, J.H. Shapiro, K. Schwab, Team Leads, Quantum Communication, Sensing and Measurements in Space, Keck Institute for Space Studies 2012

30. http://www.asterank.com/3d/

31. http://www.gdansk.karmelici.pl/zegar-pulsarowy-250

32. https://www.esa.int/Our_Activities/Navigation/ESA_sets_clock_by_distant_spinning_stars.

33. https://purdue.edu/impactearth/

34. G.S. Collins, H.J. Melosh, and R.A. Marcus, Earth Impact Effects Program: A Web-based computer program for calculating the regional environmental consequences of a meteoroid impact on Earth, Meteoritics \& Planetary Science 40, Nr 6, 817-840, 2005

35. T.D. Moyer, Relativistic Equations of Motion for the Generation of Ephemeries for the Planets, the EarthMoon Barycenter, the Moon, and a Space Probe, JPL Space Programs Summary 37-49, Vol III, 1966

36. O. Montenbruck, E. Gill, Satellite Orbits, Springer 2001

37. W. Nawrocki, Introduction to Quantum Metrology. The Revised SI System and Quantum Standards, Springer 2019

38. A. Einstein, Teoria względności i inne eseje, Prószyński i S-ka, Warszawa 1997. (The Theory of Relativity and Other Essays) (in Polish)

39. A. Einstein, Istota teorii względności, Prószyński i S-ka, Warszawa 1997. (The Meaning of Relativity) (in Polish)

40. J.B. Hartle „Gravity: An Introduction to Einstein's General Relativity”, 2003 by Pearson Education, Inc., publishing as Addison Wesley. 